\title{
Negative Memory Engrams in the Hippocampus Enhance the Susceptibility to Chronic Social Defeat Stress
}

\author{
(i)Tian Rui Zhang, ${ }^{1,2 *}$ (D)Amanda Larosa, ${ }^{1,2 *}$ Marie-Eve Di Raddo, ${ }^{2}$ Vanessa Wong, ${ }^{2}{ }^{\circledR}$ Alice S. Wong, ${ }^{2}$ \\ and $\odot$ Tak Pan Wong ${ }^{2,3}$ \\ ${ }^{1}$ Integrated Program in Neuroscience, McGill University, Montreal, Quebec H3A 2B4, Canada, ${ }^{2 B a s i c}$ Neuroscience Division, Douglas Hospital Research \\ Centre, Montreal, Quebec H4H 1R3, Canada, and '3Department of Psychiatry, McGill University, Montreal, Quebec H3A 1A1, Canada
}

The hippocampus has been highly implicated in depression symptoms. Recent findings suggest that the expression and susceptibility of depression symptoms are related to the enhanced functioning of the hippocampus. We reasoned that hippocampal engrams, which represent ensembles of neurons with increased activity after memory formation, could underlie some contributions of the hippocampus to depression symptoms. Using the chronic social defeat stress model, we examined social defeat-related hippocampal engrams in mice that are either susceptible or resilient to the stressor. TetTag mice were used to label social defeat-related hippocampal ensembles by LacZ. Engram cells correspond to ensembles that were reactivated by the same stressor. Compared with resilient and nonstressed control mice, susceptible mice exhibited a higher reactivation of social defeat-related LacZ-labeled cells (i.e., engram cells) in both the dorsal and ventral hippocampal CA1 regions. The density of CA1 engram cells correlated with the level of social avoidance. Using DREADD and optogenetic approaches to activate and inactivate social defeat-related CA1 engram cells enhanced and suppressed social avoidance, respectively. Increased engram cells in susceptible mice could not be found in the dentate gyrus. Susceptible mice exhibited more negative stimuli-related, but not neutral stimuli-related, CA1 engram cells than resilient mice in the dorsal hippocampus. Finally, chronic, but not a short and subthreshold, social defeat protocol was necessary to increase CA1 engram cell density. The susceptibility to chronic social defeat stress is regulated by hippocampal CA1 engrams for negative memory. Hippocampal negative memory engrams may underlie the vulnerability and expression of cognitive symptoms in depression.

Key words: depression; engrams; hippocampus; individual difference; memory; stress

Significance Statement

We provided evidence that negative memory hippocampal engrams contribute to the susceptibility to developing depressionrelated behavior after chronic social defeat stress. The activation of positive memory engrams has been shown to alleviate depression-related behaviors, while our findings reveal the pathological roles of negative memory engrams that could lead to those behaviors. Increased negative memory engrams could be a downstream effect of the reported high hippocampal activity in animal models and patients with depression. Unlike affective symptoms, we know much less about the cellular mechanisms of the cognitive symptoms of depression. Given the crucial roles of hippocampal engrams in memory formation, enhanced reactivation of negative memory engrams could be an important cellular mechanism that underlies the cognitive symptoms of depression.

\section{Introduction}

Apart from affective symptoms, such as sad mood, anhedonia, hopelessness, and low self-esteem, cognitive symptoms are com-

Received July 31, 2018; revised July 15, 2019; accepted July 19, 2019.

Author contributions: T.R.Z., A.L., and T.P.W. designed research; T.R.Z., A.L., and A.S.W. performed research; T.R.Z., A.L., M.-E.D.R., V.W., A.S.W., and T.P.W. analyzed data; T.R.Z. wrote the first draft of the paper; T.R.Z., A.L., M.E.D.R., V.W., and T.P.W. edited the paper; T.R.Z. and T.P.W. wrote the paper.

This work was supported by Canadian Institutes of Health Research 137003 and Natural Sciences and Engineering Research Council 241148. The present study used the services of the Molecular and Cellular Microscopy Platform in the Douglas Hospital Research Centre. We thank Dr. Frédéric Manseau (Dr. Sylvain Williams's laboratory), Dr. Jennifer Robinson (Dr. Mark Brandon's laboratory), and Dr. Rosemary Bagot for advice on the optogenetic experiment; mon in depression. A prominent cognitive symptom of depression is the negative bias in cognitive processing and memory formation (for review, see Disner et al., 2011; Joormann and Quinn, 2014). Depressed patients show enhanced encoding and recall of mood-congruent negative memory (Koster et al., 2010),

\footnotetext{
Ms. Moushumi Nath for characterizing the ArchT virus; and Melina Jaramillo Garcia for setting up the imaging experiments and the analysis.

The authors declare no competing financial interests.

*T.R.Z. and A.L. contributed equally to this work.

Correspondence should be addressed to Tak Pan Wong at takpan.wong@mcgill.ca.

https://doi.org/10.1523/JNEUROSCI.1958-18.2019

Copyright $\odot 2019$ the authors 
less forgetting of negative memory (Hertel and Gerstle, 2003), and impaired recall of positive memory (Gaddy and Ingram, 2014). Rumination, which is related to repetitive recall of negative memory (Lyubomirsky et al., 1998), is also common in depression (Nolen-Hoeksema, 2000). Increasing findings suggest that biases in cognitive processing in depression could be associated with changes in the hippocampus, a brain region that is known for its role in memory formation (Squire, 1992).

The hippocampus has long been implicated in the manifestation of depression symptoms. Meta analyses have revealed reduced hippocampal volume in depressed patients (Videbech and Ravnkilde, 2004; McKinnon et al., 2009). Therapeutic effects of classical (e.g., fluoxetine) and fast-acting (e.g., ketamine) antidepressants have been associated with hippocampal neurogenesis (Santarelli et al., 2003) and altered hippocampal glutamate receptor function (Maeng et al., 2008; El Iskandrani et al., 2015), respectively. Recent findings suggest that increased hippocampal function could contribute to the biased cognitive processing in depression. Imaging studies revealed increased hippocampal responses to sad faces (Fu et al., 2004) and stronger hippocampusamygdala connectivity during negative information encoding (Hamilton and Gotlib, 2008) in depressed patients. Moreover, attenuated hippocampal responses to negative stimuli could be induced by antidepressants (Mayberg et al., 2000; Fu et al., 2004) and observed in remitted depressed patients (Thomas et al., 2011). Using chronic social defeat stress (CSDS) as an animal model for depression-related behaviors (Krishnan et al., 2007), the expression of these behaviors has been associated with increased activity of the ventral hippocampal dentate gyrus (DG) region (Anacker et al., 2018). Suppressing ventral hippocampal glutamatergic inputs to the nucleus accumbens can enhance stress resilience of this model (Bagot et al., 2015). Increased hippocampal activity could affect the formation of engrams, which are ensembles of neurons that showed increased activity during memory formation and recall (Josselyn et al., 2015; Tonegawa et al., 2015). Apart from being a substrate for memory, hippocampal engrams have been associated with the expression of depressionrelated behaviors, so that reactivating positive memory-related hippocampal engrams can induce antidepressant effects (Ramirez et al., 2015). Increased hippocampal activity could also facilitate the formation and enhance the activity of negative memory engrams. Whether negative memory engrams contribute to the expression of depression-related behaviors warrants further investigations.

In the current study, we investigated the formation and activity of social defeat-related hippocampal engrams in mice that were stressed under the CSDS protocol. We used TetTag mice to tag hippocampal neurons that were activated by social defeat with LacZ (Reijmers et al., 2007). Engram cells were examined and defined as those LacZ labeled cells that were reactivated by the same stressor. The CSDS model allows us to separate mice according to their individual differences in stress susceptibility. We found that mice that were susceptible to CSDS had more social defeat-related engram cells in the hippocampal CA1 region than nonstressed control mice and mice that were resilient to this stressor.

\section{Materials and Methods}

Animals. Male TetTag mice were obtained from the The Jackson Laboratory (stock \#008344) (Reijmers et al., 2007). Bitransgenic TetTag mice with a C57 background carry a $c$ Fos-driven tetracycline-controlled transactivator (tTA) protein construct and a tetracycline-responsive regulatory element (tet $\mathrm{O}$ )-driven $\beta$-galactosidase ( $\mathrm{LacZ}$ ) construct. The $c F o s$ promoter can be activated by neuronal activity. This strain has been used for labeling activated neurons by the expression of LacZ via a doxycycline off (Dox-off) mechanism as previously described (Reijmers et al., 2007). Double-hemizygote TetTag mice were bred with WT C57 mice (Charles River). Only male double-hemizygote offspring (approximately oneeighth of all offspring) were used in this study. Breeding pairs and offspring were fed with Dox-containing food ( $40 \mathrm{mg} / \mathrm{kg}$, Envigo) ad libitum in a $12 \mathrm{~h} \mathrm{light/dark} \mathrm{cycle} \mathrm{(light} \mathrm{on} \mathrm{from} \mathrm{8:00} \mathrm{A.M.} \mathrm{to} \mathrm{8:00} \mathrm{P.M.).} \mathrm{LacZ}$ labeling can be induced by feeding TetTag mice with Dox-free food (Dox off), which allows the $c F o s$-driven expression of tTA to activate the tet $O$ $L a c Z$ construct. The activation of tet $O$ during Dox off also triggered the expression of a tetracycline-insensitive tTA (with a H100Y point mutation), which sustained the expression of LacZ, even after the reintroduction of Dox to maintain long-term labeling of activated neurons. The average age of the mice was 3 months. Offspring of TetTag mice that expressed only the $c F o s-t T A$ construct were used in the DREADD experiment (see below). Finally, male retired breeders of the CD1 strain (Charles River) were used for defeating mice of C57 strains. All experiments were approved by the Facility Animal Care Committee at Douglas Hospital Research Centre and followed the guidelines from Canadian Council on Animal Care (protocol 2010-5935).

Viral vectors. AAV-PTRE-tight-hM3Dq-mCherry was a gift from William Wisden (Addgene, plasmid \#66795; http://n2t.net/addgene:66795; RRID:Addgene, _66795) (Zhang et al., 2015). AAV-RAM-d2TTA::TREArchT-WPREpA (Addgene, plasmid \#84472; http://n2t.net/addgene: 84472; RRID:Addgene,_84472) and AAV-RAM-d2TTA::TRE-EGFPWPREpA (Addgene, plasmid \#84469; http://n2t.net/addgene:84469; RRID:Addgene, _84469) were gifts from Yingxi Lin (Sørensen et al., 2016). Virus was injected into the dorsal CA1 region using the following coordinates from bregma: AP -2.06 ; lateral \pm 1.40 ; DV -1.50 .

CSDS. TetTag mice were defeated by male retired breeders of the CD1 strain during social defeat. Resident CD1 mice were housed in a partitioned compartment of a rat cage, like the type of cage used for habituation, before social defeat. Each CD1 mouse was screened for its aggressiveness by attacking intruders, and only those with a $<60$ s latency were selected. The CSDS paradigm consisted of 8 episodes of defeat. In each defeat episode, a CD1 mouse was allowed to attack a TetTag mouse for up to 12 attacks in a maximum period of $5 \mathrm{~min}$. Following each social defeat episode, each TetTag mouse was housed next to the CD1 mouse in the neighboring compartment separated by a perforated partition for $24 \mathrm{~h}$. Without physical contacts, TetTag mice were stressed during cohousing by the presence of visual and odor stimuli from the CD1 mouse. Each TetTag mouse was paired with a new CD1 mouse in each of the 8 episodes of social defeat to prevent reduced number of attacks due to repeated cohousing. Control nonstressed mice, which were only handled and weighted daily, were pair-housed in neighbor partitions in a rat cage for $8 \mathrm{~d}$. After 8 daily episodes of defeat or pair-housing, social behavior of stressed and control mice was examined by the Social Interaction (SI) test.

SI test. The SI test consisted of two 150-s-long sessions of exploration in a Plexiglas open field $(44 \mathrm{~cm} \times 44 \mathrm{~cm})$. An empty perforated enclosure $(10 \mathrm{~cm} \times 5 \mathrm{~cm} \times 30 \mathrm{~cm})$ was placed in the center of the north side of the open field during the first open field session. After the end of the first open field session, a CD1 mouse was put into the enclosure before the second open field session began. Both open field sessions were performed under ambient red light, with static white noise at $60 \mathrm{~dB}$. Time spent in the interaction zone $(10 \mathrm{~cm}$ around the enclosure) during the first (empty) and second (with a CD1 mouse) open field sessions were estimated from recorded videos of these sessions using the software TopScan LITE (Clever System). The SI ratio was calculated by dividing the time mice spent in the interaction zone in the second open field session with the time they spent in the interaction zone in the first open field session. We also measured time TetTag mice spent in the two corners zones (10 $\mathrm{cm} \times 10 \mathrm{~cm}$ ) on the opposite side of the enclosure, which were farthest away from the enclosure. Corner ratios were calculated by dividing time TetTag mice spent in those corners in the second open field session by the time they spent there in the first session. Susceptible mice were defined as animals having a social interaction ratio of $<1$, indicating they spent less time in the interaction zone when a CD1 mouse was present. Resilient mice were defined as having a social interaction ratio of $>1$ and spent at 
A

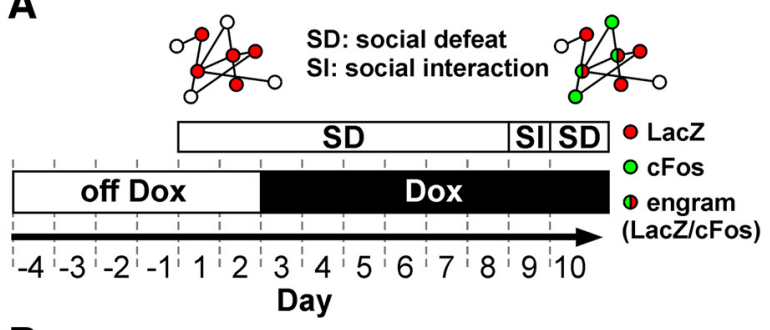

B
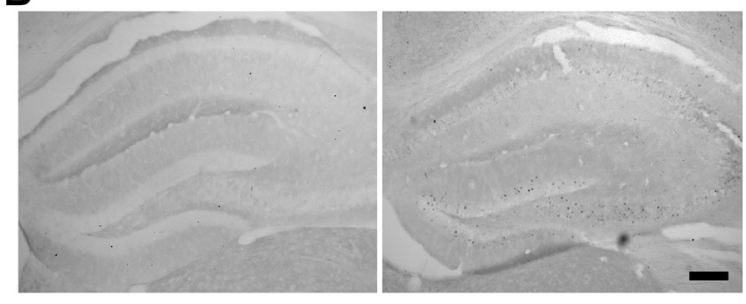

C
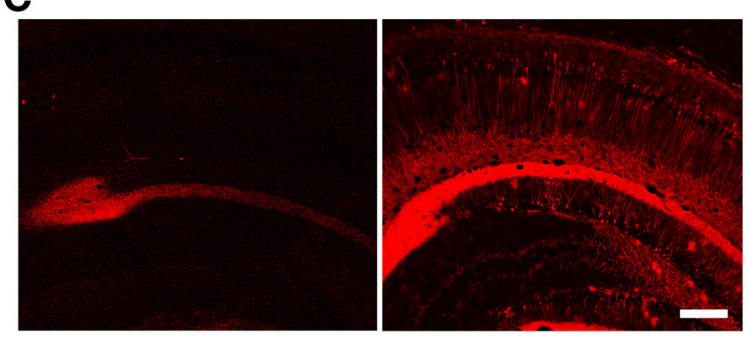

D

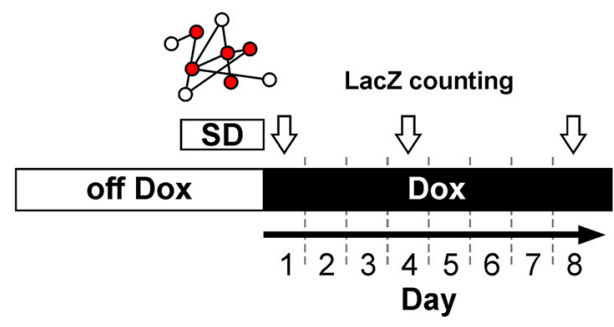

E

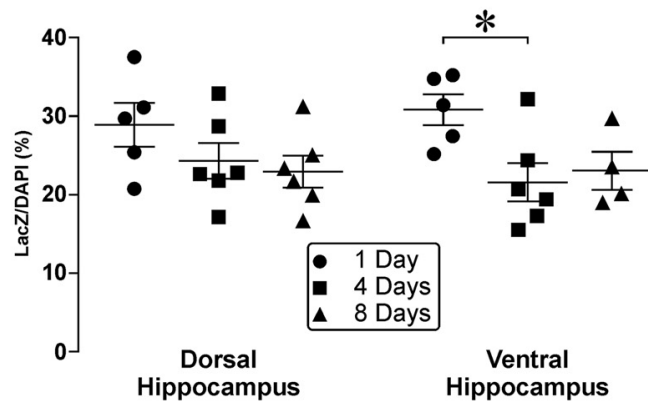

Figure 1. Social defeat (SD) triggers the formation of hippocampal ensembles. A, A schematic diagram of the experimental design. TetTag mice were off Dox for $4 \mathrm{~d}$. After two episodes of SD on day 1 and 2, labeling was blocked by putting mice on Dox-containing food (Dox). Mice were then stressed by 6 more episodes of SD. The interaction between TetTag mice and aggressors of the CD1 strain was examined in a social interaction (SI) test. One day after the SI test, mice underwent one more episode of SD to trigger ensemble reactivation. Mice were killed $90 \mathrm{~min}$ after the last episode of SD. Diagrams above the experimental plan represent the labeling of activated neurons during the first two episodes of SD (red, LacZ), during the last episode of SD (green, cFos), and engram cells that expressed both signals (red/green). B, Left, cFos-stained dorsal hippocampal sections from a control mouse that was housed in its home cage. Right, cFos-stained dorsal hippocampal sections from another control mouse that was defeated by 2 SD episodes. Scale bar, $200 \mu \mathrm{m}$. C, LacZ staining of ventral hippocampal neurons from TetTag mice that were off Dox during labeling (Dox off, right). A stained section from a mouse that was on Dox during labeling was shown on the left (Dox on). Apart from nonspecific staining near the hippocampal fissure, LacZ cells and processes cannot be found in tissue from the Dox on mouse. Scale bar, $200 \mu \mathrm{m}$. D, A schematic diagram of the experimental design for testing the stability least $50 \mathrm{~s}$ in the social interaction zone during the second open field session.

Ensemble reactivation. After the SI test, both stressed and control mice were housed singly in mouse cages. To reactivate ensembles that were related to social defeat in stressed mice, we gave stressed mice an extra episode of social defeat, followed by cohousing in the neighbor compartment with a CD1 mouse in a partitioned rat cage for 90 min to trigger cFos expression. Ensembles related to contextual information of the rat cage were reactivated in control mice to express cFos by cohousing them with another control mouse in neighbor compartments of a partitioned rat cage for $90 \mathrm{~min}$. After ensemble reactivation, mice were anesthetized and perfused by heparin-containing PBS and 4\% PFA. Brains were extracted from the skulls, postfixed in PFA overnight, and cryoprotected in $30 \%$ sucrose-containing PBS.

Immunohistochemistry. Unless indicated otherwise, all reagents were obtained from Sigma-Aldrich. Fixed brains were snap-frozen in dry icechilled isopentane before being cut into $35-\mu \mathrm{m}$-thick sections using a cryostat (Leica Microsystems). Brain sections were washed with PBS (five 5 min washes; a similar washing procedure was used between all antibody incubations), followed by a $30 \mathrm{~min}$ incubation in $0.3 \% \mathrm{NaBH}_{4}$ to quench endogenous fluorescence. After PBS washes, sections were incubated for $1 \mathrm{~h}$ in a blocking solution (3\% normal goat serum and $0.1 \%$ Triton in PBS [PBS-T]; this blocking solution was also used for diluting antibodies). For triple immunofluorescent staining, sections were incubated overnight at $4^{\circ} \mathrm{C}$ with the first primary antibody (mouse monoclonal LacZ antibody, 1:2000, MP Biomedicals, 08633651). The next day, sections were washed by PBS-T and incubated with the first secondary antibody (donkey anti-mouse Alexa-674 antibody, 1:2000, Abcam, Ab150107) for $3 \mathrm{~h}$ at room temperature. In the same fashion, incubations were done for the second primary antibody (rabbit polyclonal cFos antibody, 1:40,000, Sigma-Aldrich, F137) and the corresponding secondary antibody (goat anti-rabbit Alexa-488 antibody, 1:4000, Invitrogen, A11034). Finally, sections were incubated with 600 nM DAPI (Invitrogen, D3571) for $10 \mathrm{~min}$. Triple-labeled sections were mounted on a slide, covered with VectaShield antifade mounting medium (Vector Laboratories), and sealed with nail polish. The stained sections were scanned using a slide scanner (Olympus VS120) with the VS-ASW acquisition software to a magnification of $20 \times$ with $1115-\mu$ m-thick $z$ sections. Sections were stitched together by the VS-ASW software (Olympus).

For DAB staining of cFos, after primary antibody incubation and washes, slices were incubated for $1 \mathrm{~h}$ with a biotinylated goat anti-rabbit antibody (1:500, Vector Laboratories, BA-1000), followed by an hourlong incubation with the $\mathrm{ABC}$ reagent (1:250, Vector Laboratories, PK$7200)$. Sections were finally incubated with $\mathrm{DAB}(0.06 \%)$ and $\mathrm{H}_{2} \mathrm{O}_{2}$ for 2 min to visualize staining.

Cell counting. Analysis of the digital slides from the slide scanner were done manually with the help of Fiji (ImageJ). As there are regional differences in inputs, projections, and functions between dorsal and ventral and hippocampus (Fanselow and Dong, 2010), cell counting was performed in both regions. For CA1 counting, a $400 \mu \mathrm{m}$ (width) $\times 200 \mu \mathrm{m}$ (height) counting window was used for counting LacZ-, cFos-, and DAPI-labeled cells in the dorsal and ventral hippocampus. Only neurons in the stratum pyramidale were counted. Since we found no LacZ cells in the pyramidal layer of the CA2 and CA3 regions in both control and stressed mice, these hippocampal regions were excluded from further analysis. Finally, for cell counting in the DG region, due to the low number of double-labeled cells in the DG, the entire DG granule cell layer in the dorsal and ventral hippocampus in each section was counted. Unless specified otherwise, all staining data were presented as the density of

$\leftarrow$

of LacZ expression after Dox on. TetTag mice were off Dox for $4 \mathrm{~d}$. After two episodes of SD, labeling was blocked by putting mice on Dox. TetTag mice were killed 1, 4, and $8 \mathrm{~d}$ later (white arrows). $\boldsymbol{E}$, Scatter plots summarize the density of LacZ-positive neurons in the CA1 region of the dorsal and ventral hippocampus of TetTag mice at different time points after labeling (dorsal hippocampus: $1 \mathrm{~d}$ after defeat: $n=5 ; 4 \mathrm{~d}$ after defeat: $n=6 ; 8 \mathrm{~d}$ after defeat: $n=6$; ventral hippocampus: $1 \mathrm{~d}$ after defeat: $n=5 ; 4 \mathrm{~d}$ after defeat: $n=6 ; 8 \mathrm{~d}$ after defeat: $n=4$ ). ${ }^{*} p<$ 0.05 , post hoc Tukey's test in each hippocampal region after ANOVA. Mean \pm SEM. 
A
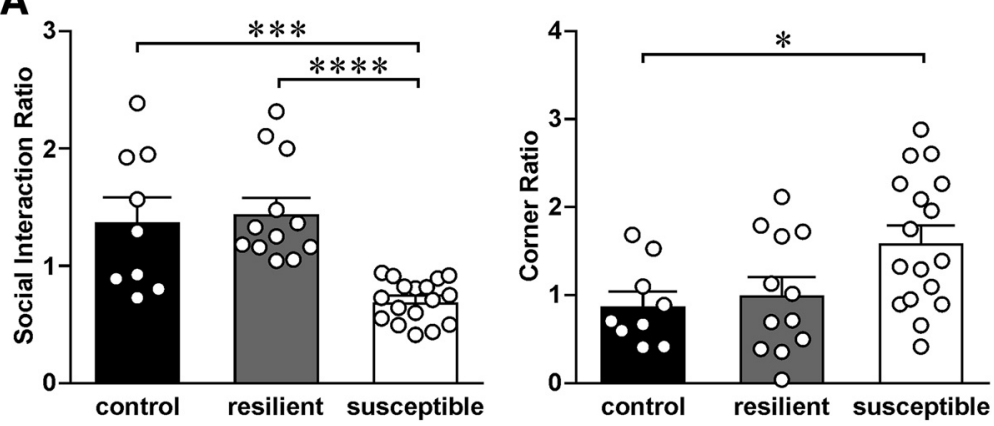

B

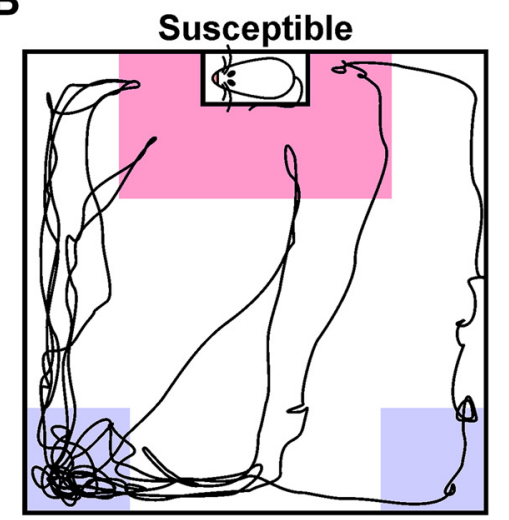

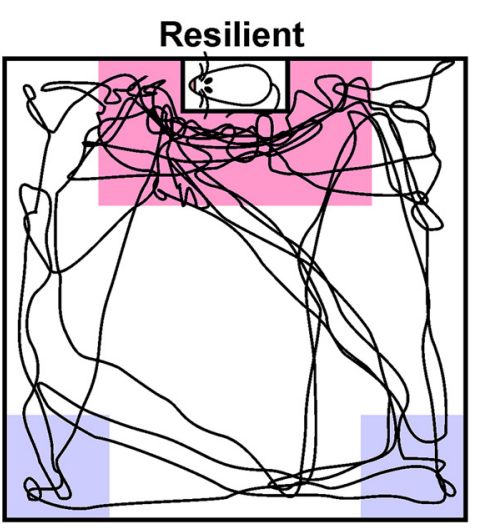

Figure 2. Susceptible, but not resilient, mice expressed social avoidance after CSDS. $A$, Histograms summarize the social interaction ratio (left) and the corner ratio (right) of susceptible $(n=17)$, resilient $(n=12)$, and nonstressed control mice $(n=$ 9). Post hoc Tukey's test after ANOVA: ${ }^{*} p<0.05 ;{ }^{* * *} p<0.001 ;{ }^{* * * *} p<0.0001$. Mean \pm SEM. $B$, Example tracks of a susceptible (left) and a resilient mouse during the second open field session of the SI test. Pink and purple zones represent the virtual interaction and corner zones, respectively. Note the cluster of tracks in the interaction and corner zones for the resilient and susceptible mice, respectively.

single-labeled (LacZ or cFos) or double-labeled (LacZ and cFos) cells by dividing their numbers with the number of DAPI-labeled cells in the counting window. Exceptions are the density of DAPI-labeled cells (all DAPI-labeled cells in the counting window) and the normalized engram cells (density of engram cells/density of LacZ- or cFos-labeled cells). To compare the density of DAPI cells in the DG between animal groups, we controlled for the differences in the size of DG between sections by normalizing the density of DAPI cells by the length of the granule cell layer. Three to five sections from each hippocampal region of each mouse were used for counting. Data from these sections were averaged, and only the mean densities of single- and double-labeled cells of each mouse were used for statistical analysis.

Experimental design and statistical analysis. All statistical analyses were performed using Prism 7 (GraphPad). Normality of data was examined by the Shapiro-Wilk test. All data were presented as mean \pm SEM.

Experiment 1: Social defeat-related hippocampal engrams in mice with different stress susceptibilities. Adult male TetTag mice were off Dox for $4 \mathrm{~d}$ during habituation before being stressed by 8 episodes of social defeat (see Fig. 1A). Habituation has been shown to reduce the labeling of hippocampal neurons from being housed in a novel environment (Radulovic et al., 1998). During habituation, 2 TetTag mice were housed in neighboring compartments of a rat cage. We found that 2 social defeat episodes were sufficient to induce cFos (see Fig. $1 B$ ) and LacZ expression (see Fig. 1C) in the hippocampus. LacZ labeling was therefore stopped after two episodes of social defeat by Dox $(1 \mathrm{~g} / \mathrm{kg})$ for $1 \mathrm{~d}$, followed by regular Dox food $(40 \mathrm{mg} / \mathrm{kg}$ ) to prevent further LacZ labeling. There was no neuronal LacZ expression in the hippocampus of TetTag mice that were always on Dox (see Fig. 1C). While the expression of LacZ decreased in the first few days after resuming the Dox diet, we observed stable LacZ expression up to $8 \mathrm{~d}$ after social defeat (see Fig. $1 D, E$ ). After a total 8 episodes of social defeat, TetTag mice were examined by the SI test. One day after the SI test, social defeat-related ensembles of stressed mice were reactivated by another defeat episode. Control TetTag mice were treated similarly as stressed mice, but they were only handled after habituation and during ensemble reactivation. Mice were killed $90 \mathrm{~min}$ after ensemble reactivation for immunostaining. There were 29 stressed and 9 control mice in this experiment. Stressed mice were furthered divided into susceptible and resilient mice according to their SI ratios (see Fig. 2). Due to the distinct roles of the dorsal and ventral hippocampus in spatial and emotional functions, data from dorsal and ventral hippocampus were separately compared. Density of LacZ, cFos, engram, and DAPI cells was separately compared between the three mouse groups using one-way ANOVA and post hoc Tukey's test (see Figs. 4, 8).

Experiment 2: Impact of activating social defeat-related engrams on social interaction. Adult male $c$ Fos- $t$ TA mice were on Dox while they were bilaterally injected into the dorsal hippocampus with $0.5 \mu \mathrm{l} \mathrm{AAV-PTRE-tight-}$ hM3Dq-mCherry (Zhang et al., 2015). One week after virus injection, they were off Dox while they were habituated in pairs in partitioned rat cages. Some mice $(n=22)$ were stressed by a short and subthreshold social defeat protocol, which consists of only two episodes of social defeat described in Experiment 1. Other mice $(n=16)$ served as controls and were only weighed and handled daily for $2 \mathrm{~d}$. One day after defeat or handling, mice were examined by the SI test. One hour before the SI test, stressed and control mice were randomly selected to receive intraperitoneal injection of either saline or clozapine- $\mathrm{N}$ oxide (CNO, $3 \mathrm{mg}$ / $\mathrm{kg})$. Mice were killed and perfused after the SI test to examine virus expression, only data from mice that show expression of DREADD (mCherry) in the CA1 region were used ( 5 mice were removed). SI ratios from these four mouse groups were compared using two-way ANOVA and post hoc Tukey's test (see Fig. 7).

Experiment 3: Impact of inactivating social defeat-related engrams on social interaction. Adult male C57 mice were on Dox while they were bilaterally injected into the dorsal hippocampus with $0.5 \mu$ l AAV-RAMd2TTA::TRE-ArchT-WPREpA or AAV-RAM-d2TTA::TRE-EGFPWPREpA (Sørensen et al., 2016). A week later, fiber-optic cannulas, which were constructed in our laboratory using a short segment of multimode optical fiber ( $200 \mu \mathrm{m}$ diameter, $0.39 \mathrm{NA}$ ) and a ceramic ferrule (1.25 mm diameter, $230 \mu \mathrm{m}$ bore size), were implanted above the CA1 region of the dorsal hippocampus of virus injected mice (coordinates from bregma: AP: -2.06 ; lateral: \pm 1.40 ; DV: -1.30 ). One week after fiber-optic cannulas implantation, mice were off Dox while they were habituated in pairs in partitioned rat cages. Some mice $(n=10)$ were stressed by the standard CSDS protocol, which consists of 8 episodes of social defeat described in Experiment 1. Other mice $(n=12)$ served as controls and were only weighed and handled daily for $8 \mathrm{~d}$. One day after defeat or handling, mice were attached by two patch cables to continuously deliver $520 \mathrm{~nm}(15 \mathrm{~mW})$ green laser to their dorsal hippocampi during the second session of SI test (with a CD1 mouse in the enclosure). Mice were killed and perfused after the SI test to examine virus expression. Only mice with virus expression and proper placement of the optic fibers in the CA1 region were included in the analysis (3 mice were removed). SI ratios from these 4 mouse groups were logtransformed and compared using two-way ANOVA and post hoc Tukey's test (see Fig. 7).

Experiment 4: Neutral contextual stimuli-related hippocampal engrams in mice with different stress susceptibilities. To label neutral contextual stimuli-related engrams, adult male TetTag mice were off Dox for $6 \mathrm{~d}$ during habituation before being stressed by 8 episodes of social defeat 


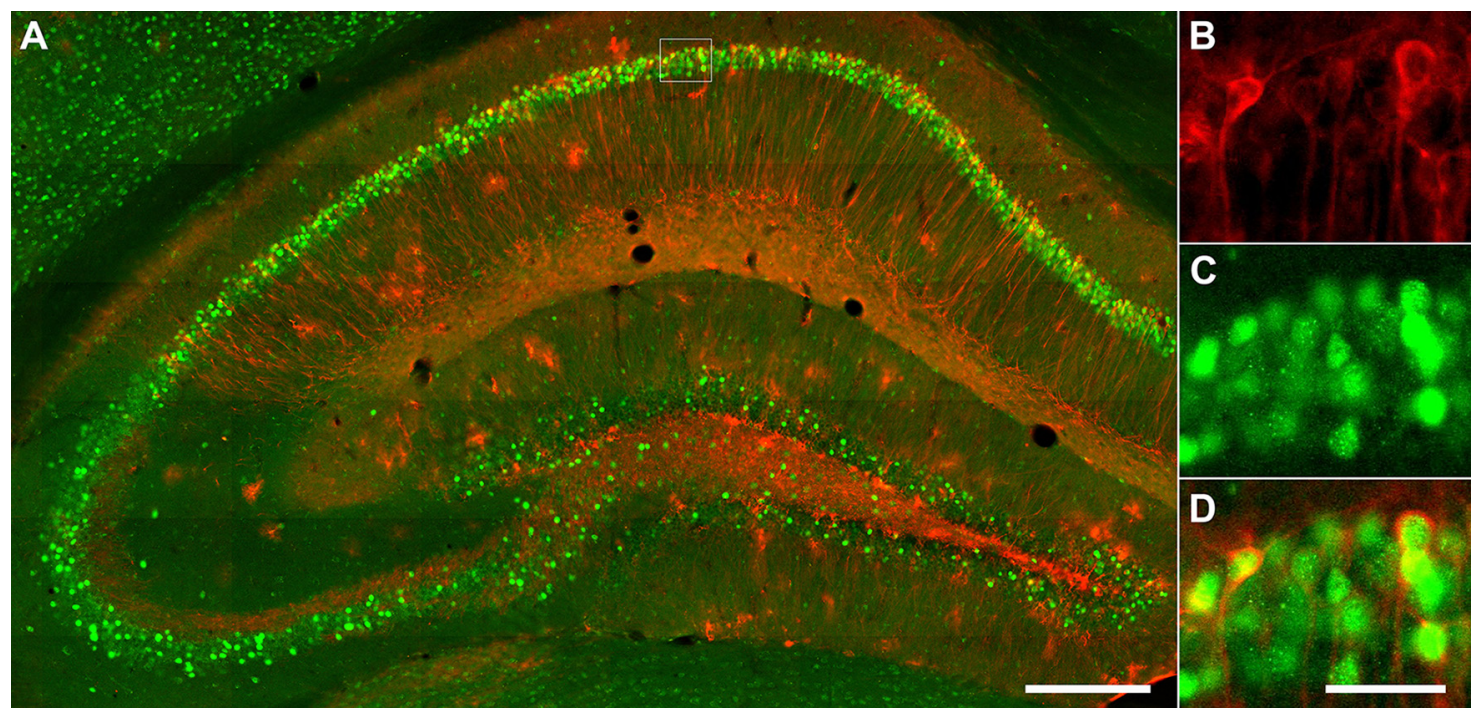

Figure 3. Hippocampal LacZ and cFos staining of a TetTag mouse. A, Fluorescent micrographs of dorsal hippocampal CA1 neurons that were stained for LacZ (red) and cFos (green). Part of the CA1 (dotted line square) was enlarged to show $\operatorname{LacZ}(\boldsymbol{B}), \mathrm{CF}$ os ( $\boldsymbol{C}$, and the overlapping of LacZ and cFos in engram cells (D). Scale bars: $\boldsymbol{A}, 250 \mu \mathrm{m} ; \boldsymbol{B}-\boldsymbol{D}, 40 \mu \mathrm{m}$.

(see Fig. 9A). LacZ expression was arrested $1 \mathrm{~d}$ before social defeat by 1 $\mathrm{g} / \mathrm{kg}$ Dox, followed by $40 \mathrm{mg} / \mathrm{kg}$ Dox throughout the 8 episodes of social defeat. One day after the last episode of defeat, TetTag mice were examined by the SI test, followed $1 \mathrm{~d}$ later with the reactivation of ensembles by another episode of social defeat. Control TetTag mice were treated similarly as stressed mice but were only handled after habituation and during ensemble reactivation. Mice were killed $90 \mathrm{~min}$ after ensemble reactivation for immunostaining. We had a total of 34 stressed and 19 control mice. Stressed mice were furthered divided into susceptible and resilient mice according to their SI ratio. Due to the distinct roles of the dorsal and ventral hippocampus in spatial and emotional functions, data from the dorsal and ventral hippocampus were separately compared. Density of LacZ, cFos, engram, and DAPI cells was separately compared between the three mouse groups using one-way ANOVA and post hoc Tukey's test (see Fig. 9).

Experiment 5: Impact of subthreshold social defeat on hippocampal engrams. Adult male TetTag mice were off Dox for $4 \mathrm{~d}$ during habituation before being stressed by two episodes of social defeat (see Fig. 10A). LacZ labeling was stopped by putting mice on Dox after the second social defeat episode. Stressed mice were examined by the SI test either $1 \mathrm{~d}$ ( $n=$ 8; defeated no delay) or $7 \mathrm{~d}(n=9$; defeated with delay) after social defeat, followed by the reactivation of ensembles $1 \mathrm{~d}$ after the SI test by another social defeat episode. Control TetTag mice $(n=8)$ were treated similarly as stressed mice but were only weighed and handled after habituation and during ensemble reactivation. Mice were killed 90 min after ensemble reactivation for immunostaining. Data from the dorsal and ventral hippocampus were separately compared. Density of LacZ, cFos, engram, and DAPI cells was separately compared between the three mouse groups using one-way ANOVA and post hoc Tukey's test (see Fig. 10).

\section{Results}

Using the CSDS protocol (Fig. 1A), we identified 17 susceptible mice that displayed social avoidance (i.e., SI ratio $<1$ ) and 12 resilient mice that showed normal social behavior after stress (Fig. $2 A, B$ ). In addition, 9 control nonstressed mice were habituated and fed with Dox and normal food like the stressed mice. These mice were pair-housed with another TetTag or nontransgenic littermates for $8 \mathrm{~d}$ after habituation and were only handled daily. One-way ANOVA revealed a significant difference in SI ratio between animal groups $\left(F_{(2,35)}=15.7 ; p=1.37 \mathrm{E}-05\right)$ with susceptible mice having lower SI than control (post hoc Tukey's test: $p=5.24 \mathrm{E}-04)$ and resilient mice $(p=4.28 \mathrm{E}-05)$.
We also examined the time mice spent in the corners of the open field during the SI tests (Fig. 2A,B). Susceptible mice spent significantly more time in corner zones than control and resilient mice in the second open field session when a social object was present in the enclosure. Comparing the corner ratios revealed a significant between group difference $\left(F_{(2,35)}=4.56 ; p=0.0173\right)$. The corner ratio of susceptible mice was significantly higher than control $(p=0.0319)$. Although susceptible and resilient mice displayed distinct behaviors during the SI test, we did not observe differences in the number of attacks ( $11.8 \pm 0.2$ for susceptible mice vs $11.5 \pm 0.3$ for resilient mice) and the duration of social defeat (i.e., time used for all attacks: $153.4 \pm 13.0 \mathrm{~s}$ for susceptible mice vs $143.6 \pm 13.9 \mathrm{~s}$ for resilient mice) between these two groups. Finally, all three mouse groups showed similar weight gain before and after social defeat $(1.44 \pm 0.30 \mathrm{~g}$ for control mice; $1.85 \pm 0.34 \mathrm{~g}$ for resilient mice; $1.38 \pm 0.39 \mathrm{~g}$ for susceptible mice).

\section{Susceptible mice displayed more engram cells in the} hippocampal CA1 region than resilient and control mice

To find out whether stress susceptibility is related to the reactivation of hippocampal ensembles that were labeled during social defeat, we examined the reactivation of LacZ ensembles that were formed during the first two episodes of social defeat by an extra episode of social defeat $1 \mathrm{~d}$ after the SI test in stressed mice (Fig. $1 A$ ). After $90 \mathrm{~min}$ following the extra episode of social defeat, stressed mice were killed for immunostaining of LacZ and cFos to reveal activated ensembles. Control mice were only exposed to the context during LacZ expression (i.e., pair-housed in a partitioned rat cage) for $90 \mathrm{~min}$ to examine the reactivation of neutral context-related LacZ ensembles. Reactivated engram cells in ensembles were represented by double-labeled cells that expressed both LacZ and cFos (Fig. 3; also see Fig. 5). The formation of these double-labeled engram cells cannot be explained by probabilistic reasons because the density of double-labeled cells in all mouse groups was significantly higher than chance (LacZ/DAPI $\times$ cFos/ DAPI) in both the dorsal (control: $t_{(8)}=4.23, p=2.87 \mathrm{E}-03$; resilient: $t_{(11)}=7.63, p=1.03 \mathrm{E}-05$, susceptible: $t_{(15)}=7.45, p=$ $2.06 \mathrm{E}-05$ ) and the ventral hippocampus (control: $t_{(8)}=5.10$, 
A
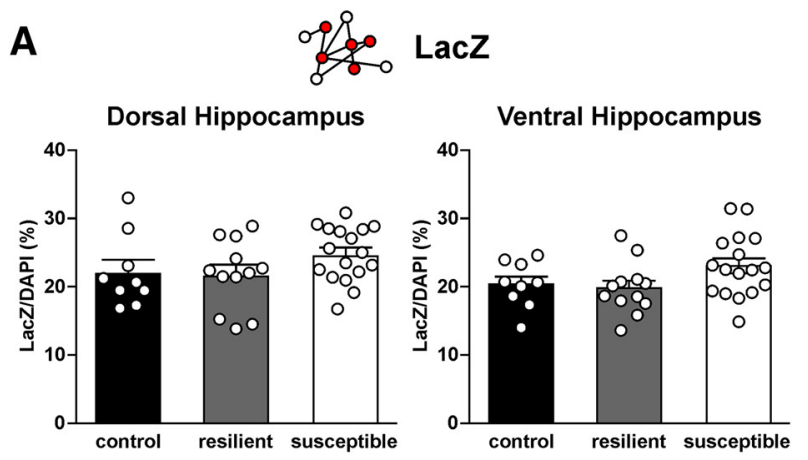

B
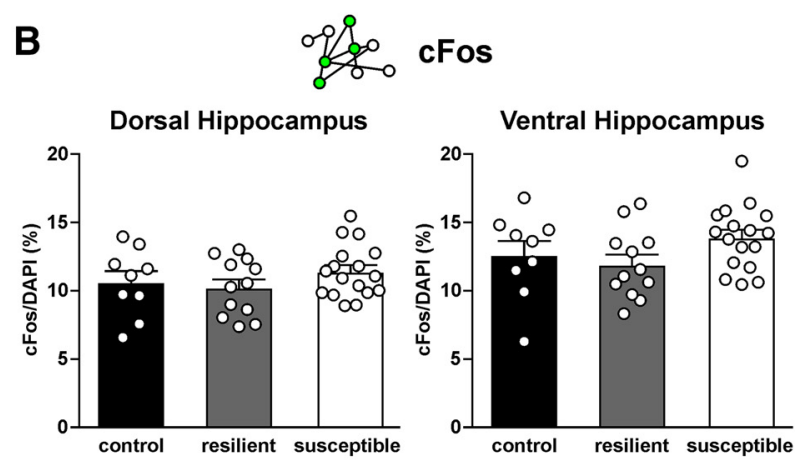

C

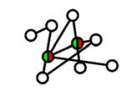

\section{Engram cells}

Dorsal Hippocampus
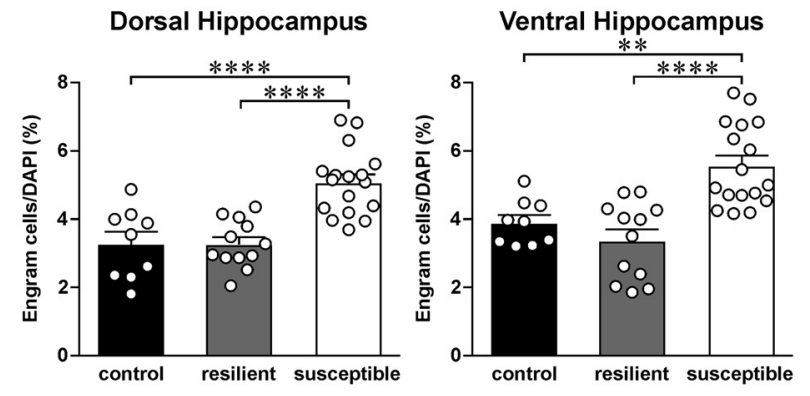

D

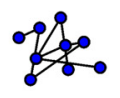

DAPI
Ventral Hippocampus

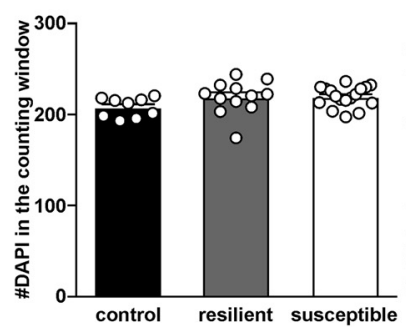

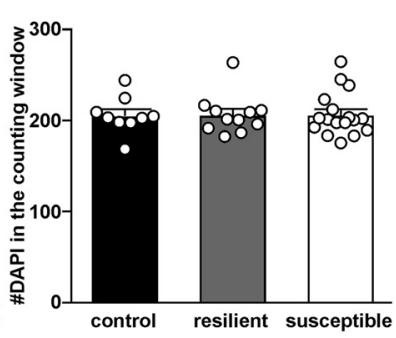

Figure 4. Expression of LacZ, cFos, and engram cells in the CA1 region of the dorsal and ventral hippocampus of control, resilient, and susceptible mice. $A$, Histograms represent the density of LacZ cells in the CA1 region of dorsal (left) and ventral hippocampus (right) of control $(n=9)$, resilient $(n=12)$, and susceptible mice $(n=17)$. Mean \pm SEM. $\boldsymbol{B}$, Histograms represent the density of cFos cells in the CA1 region of dorsal (left) and ventral hippocampus (right) of control, resilient, and susceptible mice. Mean \pm SEM. C, Histograms represent the density of engram cells (double-labeled for both LacZ and CFos) in the CA1 region of dorsal (left) and ventral hippocampus (right) of control, resilient, and susceptible mice. Tukey's test after ANOVA: ${ }^{* *} p<0.01 ;{ }^{* * *} p<0.0001$. Mean \pm SEM. D. Histograms represent the density of DAPI cells in the CA1 region of dorsal (left) and ventral hippocampus (right) of control, resilient, and susceptible mice. Mean \pm SEM.

$p=9.35 \mathrm{E}-04$; resilient: $t_{(11)}=3.87, p=2.62 \mathrm{E}-03$, susceptible: $\left.t_{(15)}=6.62, p=8.13 \mathrm{E}-06\right)$.

Densities of LacZ, cFos, and engram cells in the dorsal and ventral hippocampus were separately compared to reveal regionspecific differences. Although only resilient and susceptible mice were stressed by CSDS, we did not observe significant differences in the density of LacZ (Fig. 4A) and cFos cells (Fig. 4B) between the three mouse groups in both the dorsal and ventral hippocampal CA1 regions. However, we found that the density of engram cells in susceptible mice was significantly higher than control and resilient mice in both the dorsal (Fig. $4 C ; F_{(2,35)}=18.4 ; p=$ $3.54 \mathrm{E}-06$; post hoc Tukey's test: control vs susceptible, $p=8.88 \mathrm{E}-$ 05 , resilient vs susceptible, $p=2.21 \mathrm{E}-05)$ and the ventral hippocampus $\left(F_{(2,35)}=16.2 ; p=1.07 \mathrm{E}-05\right.$; post hoc Tukey's test: control vs susceptible, $p=1.79 \mathrm{E}-03$, resilient vs susceptible, $p=$ $1.52 \mathrm{E}-05)$. Since we have previously shown that CSDS has different impacts on hippocampal volume in susceptible and resilient mice (Tse et al., 2014), we asked whether changes in the density of CA1 neurons were responsible for the increase in engram cell density in susceptible mice (Fig. 4D). However, we did not observe differences in the density of DAPI CA1 cells between these mouse groups. Figure 5 shows representative images of the staining of LacZ, cFos, and DAPI in the dorsal CA1 region of control, resilient, and susceptible mice.

Although we did not observe significant changes in the density of LacZ cells between the three animal groups, when we analyzed data from the dorsal and ventral hippocampus separately, twoway ANOVA analysis of the effect of dorsal and ventral regions and the animal group on the density of LacZ cells revealed a significant effect of animal group (effect of animal groups: $F_{(2,70)}$ $=4.23, p=0.0185$ ), and a significantly higher LacZ cell density in susceptible mice than in resilient mice when both dorsal and ventral data were pooled together (post hoc Tukey's test: control vs susceptible, $p=0.104$; resilient vs susceptible, $p=0.025$ ). Similarly, two-way ANOVA of pooled dorsal and ventral data of the density of cFos cells revealed a significant animal group effect $\left(F_{(2,70)}=3.27, p=0.0438\right)$. Post hoc Tukey's test revealed a higher density of cFos cells in susceptible mice compared with resilient mice $(p=0.0401)$. The need of pooling dorsal and ventral hippocampal data together to reveal a significant group effect suggests that the increase in LacZ and cFos cell density in susceptible mice is modest. Similar changes in LacZ and cFos, which were labeled at different time points, suggest a long-lasting increase in neuronal activation in susceptible mice.

The increase in LacZ and cFos cell density may underlie the increased engram cell formation in susceptible mice. To test this, we normalized the engram cell density with the density of LacZ cells or cFos cells and compared the data between the three animal groups. After LacZ normalization, susceptible mice (24.8 \pm $0.968 \%)$ still have more engram cells than both control $(22.2 \pm$ $1.78 \%)$ and resilient mice $(21.8 \pm 1.46 \%)$ in the dorsal hippocampus $\left(F_{(2,35)}=9.06 ; p=6.72 \mathrm{E}-04\right.$; post hoc Tukey's test: control vs susceptible, $p=2.56 \mathrm{E}-03$, resilient vs susceptible, $p=$ $4.23 \mathrm{E}-03)$. Susceptible mice $(23.1 \pm 1.10 \%)$ also have more normalized engram cells than resilient mice $(19.8 \pm 1.10 \%)$ only in the ventral hippocampus $\left(F_{(2,35)}=6.78 ; p=3.26 \mathrm{E}-03\right.$; post hoc Tukey's test: control $(20.4 \pm 1.13 \%)$ vs susceptible, $p=0.0738$, resilient vs susceptible, $p=3.21 \mathrm{E}-03)$. After cFos normalization, susceptible mice $(45.0 \pm 1.93 \%)$ still have more engram cells than both control $(31.4 \pm 3.00 \%)$ and resilient mice $(32.3 \pm$ $1.56 \%)$ in the dorsal hippocampus $\left(F_{(2,35)}=14.3 ; p=2.90 \mathrm{E}-\right.$ 05; post hoc Tukey's test: control vs susceptible, $p=2.84 \mathrm{E}-04$, resilient vs susceptible, $p=2.20 \mathrm{E}-04)$. In the ventral hip- 


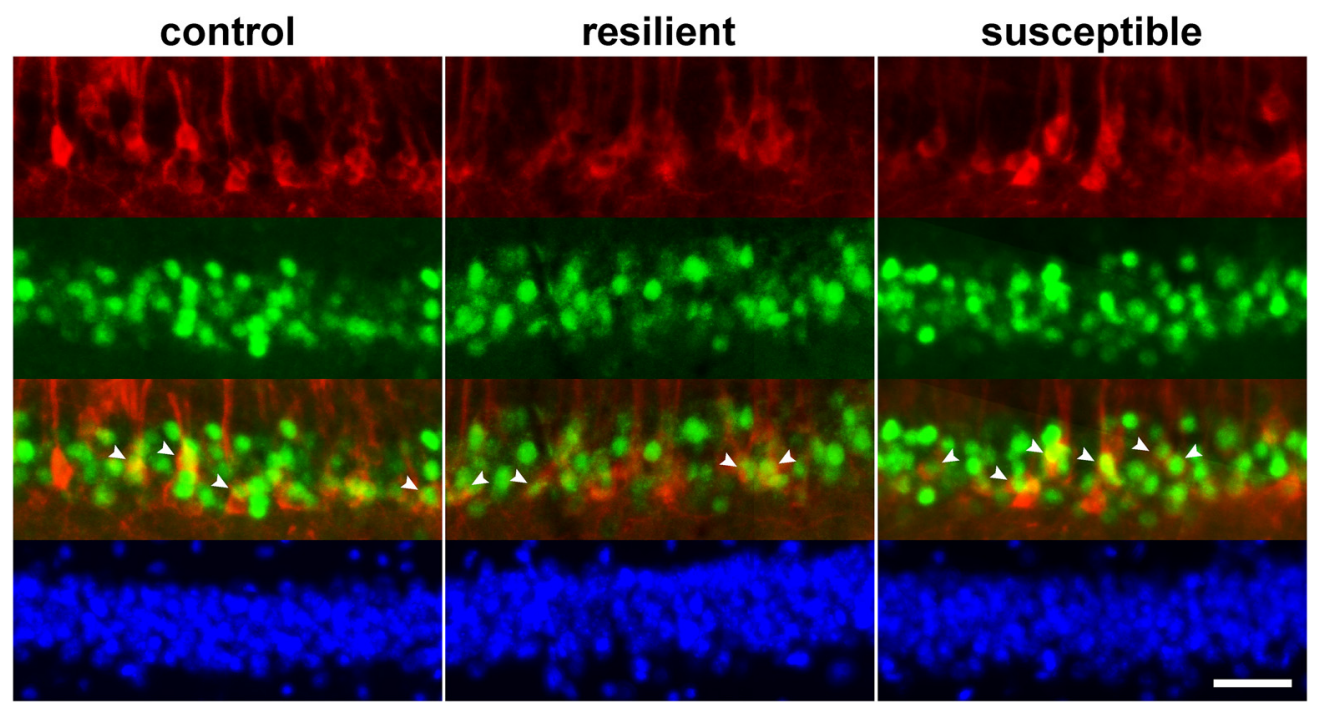

Figure 5. Engram cells in control, resilient, and susceptible mice. Representative fluorescent micrographs of dorsal hippocampal CA1 neurons that were stained for LacZ (red), cFos (green), and DAPI (blue). White arrowheads indicate engram cells with colocalized LacZ and cFos signals. Scale bars, $40 \mu \mathrm{m}$.

pocampus $\left(F_{(2,35)}=7.31 ; p=2.22 \mathrm{E}-03\right)$, susceptible mice $(40.2 \pm 1.58 \%)$ have more engram cells than resilient mice $(28.6 \pm 2.84 \%)$ but not than control mice (32.5 $\pm 3.05 \%$; post hoc Tukey's test: control vs susceptible, $p=0.0740$, resilient vs susceptible, $p=2.03 \mathrm{E}-03$ ).

The higher engram cell density in susceptible mice than resilient and control mice suggests that engram cell density is related to the expression of depression-related behavior of these mice. We separated control and stressed mice and correlated their engram cell density with their performances in the SI test. We found that CA1 engram cell density in both the dorsal (Fig. $6 A ; R^{2}=$ $0.431, p=1.10 \mathrm{E}-04$ ) and ventral hippocampus (Fig. $6 B ; R^{2}=$ $0.295, p=2.32 \mathrm{E}-03$ ) of stressed mice correlated negatively with the SI ratio. However, no significant correlation between CA1 engram cell density and SI ratio was found in control mice. When we examined the relationship between CA1 engram cell density and the corner ratio, we also found a significant correlation between dorsal CA1 engram cell density and corner ratios in stressed mice (Fig. 6C; $R^{2}=0.187, p=0.0191$ ) and between dorsal (Fig. $6 C ; R^{2}=0.446, p=0.0493$ ) and ventral CA1 engram cell density and corner ratios in control mice (Fig. $6 D ; R^{2}=$ $0.580, p=0.0171)$. Interestingly, the engram cell density of stressed and control mice correlated positively and negatively with the corner ratio, respectively. These findings suggest that high CA1 engram cell density in susceptible mice is related to the expression of social avoidance.

\section{Effect of manipulating the activity of engram cells on mouse performance in the SI test}

Findings from the correlation analyses suggest that reactivating CA1 engram cells can trigger social avoidance. To test that, we used the $c$ Fos-tTA offspring from TetTag mice that lack the LacZ construct (Fig. 7A). While these mice were fed with Dox, we bilaterally injected AAV-PTRE-tight-hM3Dq-mCherry into the dorsal hippocampi of adult $c$ Fos- $t$ TA mice (Fig. $7 B$ ). After 1 week, we put these mice off Dox for $2 \mathrm{~d}$ before stressing mice with a subthreshold social defeat protocol with only two episodes of defeat. tTA from activated neurons will bind to TRE to trigger the expression of excitatory DREADD hM3Dq in these neurons, which can be activated by a DREADD ligand CNO. $c$ Fos- $t$ TA mice that have received AAV-PTRE-tight-hM3Dq-mCherry in- jection but no social defeat served as controls. One day after the last social defeat episode, we injected stressed and control mice with either vehicle or CNO $(3 \mathrm{mg} / \mathrm{kg})$ at $1 \mathrm{~h}$ before the SI test. Two-way ANOVA of the effect of drug treatment and stress revealed a significant effect of drug treatment (Fig. $7 C ; F_{(1,34)}=$ $10.8 ; p=2.32 \mathrm{E}-03)$, no effect of stress $\left(F_{(1,34)}=0.737 ; p=0.397\right)$, and a significant interaction between drug treatment and stress $\left(F_{(1,34)}=8.08 ; p=7.51 \mathrm{E}-03\right.$ ). Pairwise comparisons (post hoc Tukey's test) revealed significant differences between the defeated CNO group and the control saline group $(p=0.0251)$; the defeated CNO group and the control CNO group $(p=5.25 \mathrm{E}$ $04)$; the defeated CNO group and the defeated saline group ( $p=$ 0.0358 ). These findings suggest the activation of social defeatrelated hippocampal CA1 engrams reduces social interaction.

If CNO promotes social avoidance by activating CA1 engram cells, inactivating CA1 engram cells that were labeled during social defeat could reduce social avoidance resulting from an $8 \mathrm{~d}$ CSDS protocol. To test this, we used the newly developed Robust Activity Marking (RAM) system to express ArchT in activated CA1 neurons during the first $2 \mathrm{~d}$ of social defeat (Fig. 7D). The RAM system consists of an engineered activity-regulated promotor that triggers tTA expression in activated neurons and a TRE domain that is driven by tTA to express effector proteins, such as ArchT or GFP. Expression of ArchT or GFP can be arrested by Dox, which prevents tTA from binding the TRE. We injected mice with AAV-RAM-d2TTA::TRE-ArchT-WPREpA or a control virus AAV-RAM-d2TTA::TRE-EGFP-WPREpA during Dox on. After mice recovered from fiber-optic cannula implantation, we allowed the expression of ArchT or GFP during the first two episodes of social defeat (see example of ArchT expression in Fig. $7 E)$. After the end of the 8-d-long standard CSDS, we examined the behavioral effect of optogenetic stimulation using a green laser $(520 \mathrm{~nm})$ during the SI test. Mice receiving the GFP control virus (i.e., expressing only GFP in activated neurons) were either handled (nonstressed) or stressed by the standard CSDS to examine the impact of virus injection and optogenetic stimulation on mouse performance in the SI test. We also added control and defeated mice that received the ArchT virus to examine the impact of inhibiting activated CA1 neurons on the SI ratio. We log-transformed the SI ratio data of this experiment into normality and compared the effects of virus expression and stress on the 
A

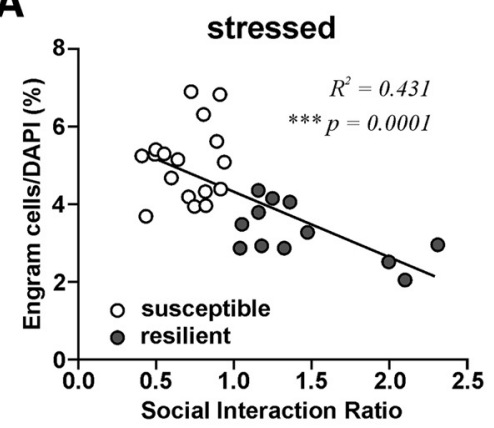

B

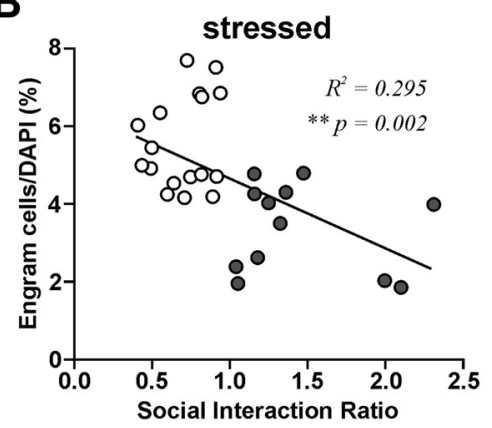

C

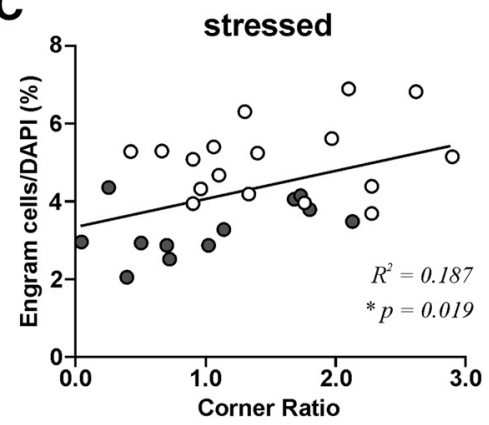

D

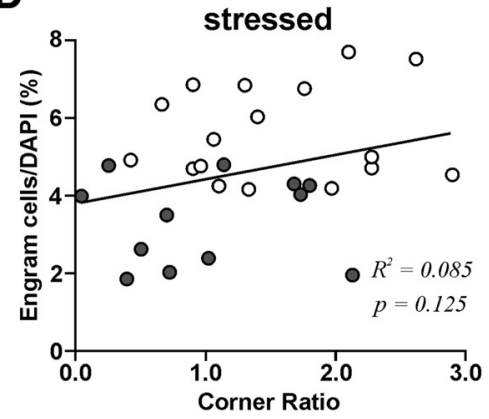

control

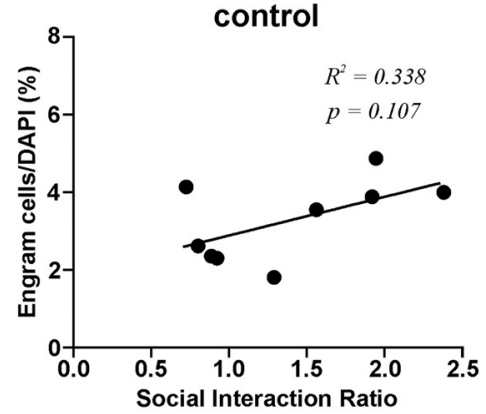

control

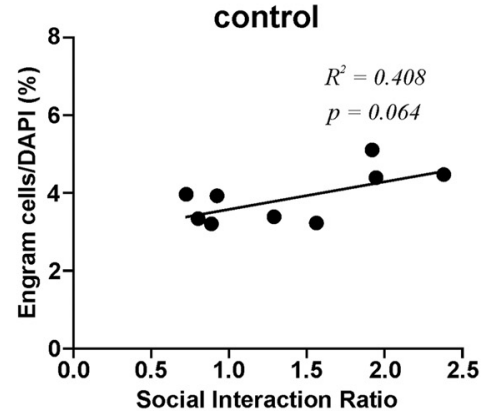

control

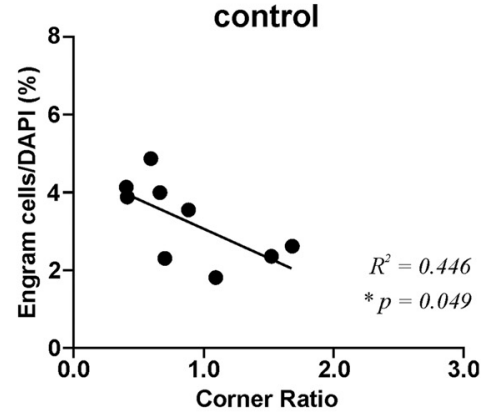

control

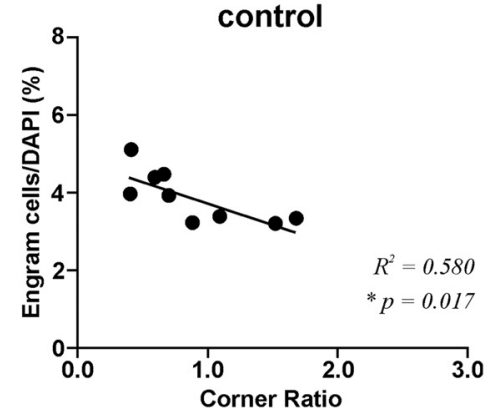

Figure 6. Density of CA1 engram cells correlates with depression-related behaviors. Scatter plots of CA1 engram cells density versus social interaction ratio of stressed (left, include both susceptible and resilient mice, $n=29$ ) and control mice (right, $n=9$ ) in the dorsal $(\boldsymbol{A})$ and ventral hippocampus $(\boldsymbol{B})$. Scatter plots of $C A 1$ engram cells density versus corner ratio of stressed and control mice in the dorsal $(\boldsymbol{C})$ and ventral $(\boldsymbol{D})$ hippocampus.

SI ratio using two-way ANOVA. We found a significant effect of virus expression (Fig. $7 F ; F_{(1,18)}=6.87 ; p=0.0191$ ), a significant effect of stress $\left(F_{(1,18)}=4.66 ; p=0.0457\right)$, and a significant interaction between virus expression and stress $\left(F_{(1,18)}=5.32\right.$; $p=0.0332)$. Post hoc comparison revealed a significant difference between GFP-expressing control and defeated mice $(p=$ 0.0348 ), a significant difference between GFP-expressing defeated mice and ArchT-expressing defeated mice $(p=0.0186)$, and a significant difference between GFP-expressing defeated

mice and ArchT-expressing control mice $(p=0.0131)$. These findings suggest that inactivating CA1 engram cells by ArchT prevents social avoidance after CSDS.

CSDS reduced engram cell density in the hippocampal DG region

Fear memory formation and recall have been associated with engrams in the DG (Liu et al., 2012; Deng et al., 2013; Denny et al., 2014). We next examined whether susceptible mice also express more DG engram cells than other mouse groups. Similar to findings we observed from the CA1 region, we did not find changes in the density of LacZ (Fig. 8A) and cFos cells (Fig. $8 B$ ) in the DG between the three mouse groups. Interestingly, we found engram cell density in control mice to be higher than both resilient and susceptible mice in the ventral DG (Fig. $8 C ; F_{(2,34)}=$ 5.59; $p=7.97 \mathrm{E}-03$; post hoc Tukey's test: control vs resilient, $p=0.0263$, control vs susceptible, $p=8.40 \mathrm{E}-03$ ). The fact that both the resilient and susceptible groups displayed similar changes in engram cell density suggests an effect due to stress. Similar to our prediction, engram cell density in the ventral DG of control mice remained higher than pooled data from the susceptible and resilient groups (control vs stressed mice: $t_{(35)}=3.33, p=$ 2.04E-03). Similarly, in the dorsal DG, we observed a trend where engram cell density in stressed mice is lower than control mice (control vs stressed mice: $t_{(36)}=$ $1.86, p=0.0717$ ). Finally, we compared the density of DAPI-labeled neurons in the DG of the three mouse groups and observed no difference between groups (Fig. 8D), even after data of susceptible and resilient mice were pooled together. DG engram cells therefore may not contribute to the susceptibility to CSDS.

Engram cell formation in susceptible mice caused by neutral stimuli

Even after habituation, we saw overlapping LacZ and cFos ensembles in control nonstressed mice. The density of engram cells was higher than the chance levels (i.e., LacZ/DAPI vs LacZ/DAPI $\times$ cFos/ DAPI; dorsal hippocampus: $t_{(8)}=4.23$, $p=2.87 \mathrm{E}-03$; ventral hippocampus: $\left.t_{(8)}=5.10, p=9.35 \mathrm{E}-04\right)$. These findings suggested that, during Dox off, the exposure to neutral contextual information triggered the formation of LacZ ensembles in the CA1 region. These ensembles were reactivated by reexposure to the same context. Engram cells observed in stressed mice in Figure 4 were likely due to the reactivation of ensembles that are related to both neutral (contextual information) and negative (social defeat) stimuli. To find out whether susceptible mice also exhibited higher reactivation of neutral stimuli-related LacZ ensembles than other mouse 
A

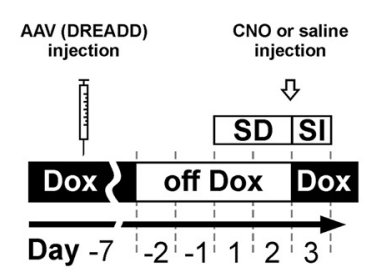

B
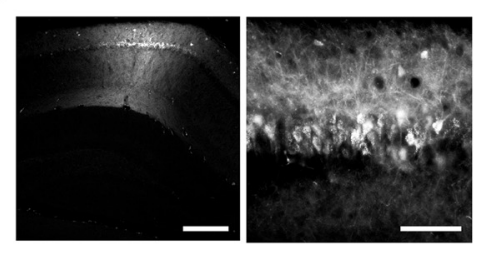

C

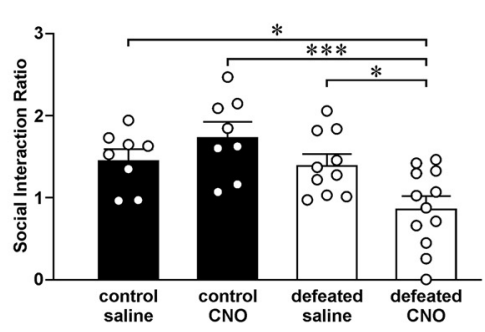

D

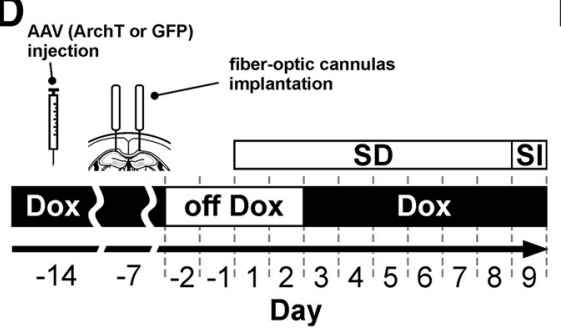

$\mathbf{E}$

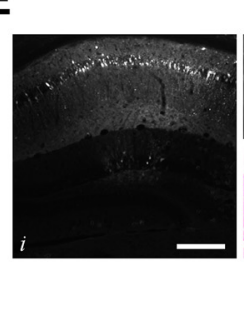

$\mathbf{F}$

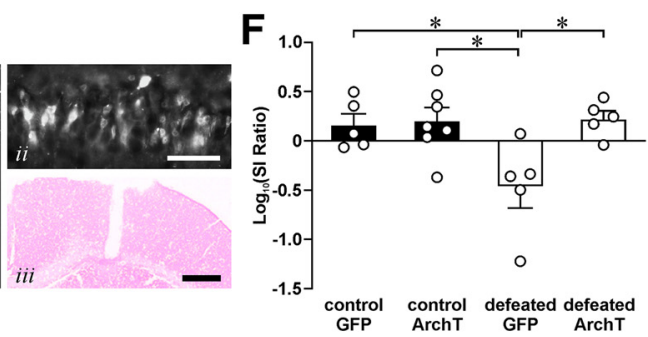

Figure 7. Effect of manipulating social defeat-related CA1 engram cells on social interaction ratio. A, A schematic diagram of the experimental design. .Fos-tTA mice were bilaterally injected with AAV-PTRE-tight-hM3Dq-mCherry into the dorsal hippocampal CA1 region. One week later, they were off Dox for $2 \mathrm{~d}$ before being stressed by a subthreshold social defeat (SD) protocol, which consisted of two episodes of SD. One day after defeat, mice were injected by either CNO $(3 \mathrm{mg} / \mathrm{kg}$ ) or saline at $1 \mathrm{~h}$ before the social interaction (SI) test. B, Fluorescent micrographs represent the expression of AAV-PTRE-tight-hM3Dq-mCherry in the dorsal hippocampal CA1 region under low magnification (left, scale bar, $200 \mu \mathrm{m}$ ) and high magnification (right, scale bar, $50 \mu \mathrm{m}$ ). C, Histograms represent the social interaction ratio of mice from different groups (control saline: $n=8$; control CNO: $n=8$; defeated saline: $n=10$; defeated CN0: $n=12$ ). Tukey's test after two-way ANOVA: ${ }^{*} p<0.05 ;{ }^{* * *} p<0.001$. Mean \pm SEM. $\boldsymbol{D}$, A schematic diagram of the experimental design. WT mice were bilaterally injected with AAV-RAM-d2TTA::TRE-ArchT-WPREpA (ArchT) or AAV-RAM-d2TTA::TRE-EGFP-WPREpA (GFP) into the dorsal hippocampal CA1 region. One week later, they were implanted with fiber-optic cannulas. After a week, they were off Dox for $2 \mathrm{~d}$ before being stressed by a chronic social defeat protocol (8 episodes of SD). Control nonstressed mice were only handled while the stressed mice were defeated. One day after the end of handling (control) or SD (defeated), mice were examined in the SI test with light on $(520 \mathrm{~nm}, 15 \mathrm{~mW}$, continuously on during the second session of social interaction with a CD1 mouse inside the enclosure). $\boldsymbol{E}$, Fluorescent micrographs represent the expression of AAV-RAM-d2TTA::TRE-ArchT-WPREpA in the dorsal hippocampal CA1 region under low magnification (Ei; scale bar, $200 \mu \mathrm{m}$ ) and high magnification (Eii; scale bar, $50 \mu \mathrm{m}$ ). Eiii, Fast red stained section shows the placement of a fiber-optic cannula (scale bar, $400 \mu \mathrm{m}$ ). $\boldsymbol{F}$, Histograms represent the log-transformed social interaction ratio of mice from different groups (GFP control: $n=5$; GFP defeated: $n=5$; ArchT control: $n=7$; ArchT defeated: $n=5$ ). Tukey tests after two-way ANOVA: ${ }^{*}<0.05$. Mean \pm SEM.

groups, we stopped LacZ labeling before social defeat and studied ensemble reactivation (Fig. 9A). Both control and stressed mice were habituated off Dox in the partitioned rat cage for $6 \mathrm{~d}$ to maintain a similar duration of LacZ expression as in previous experiments (see Fig. $4 ; 4 \mathrm{~d}$ habitation plus $2 \mathrm{~d}$ of social defeat). LacZ expression was stopped $1 \mathrm{~d}$ before social defeat by Dox, followed by similar procedures we used for CSDS and SI test in Figure $1 A$ (see Fig. 9A). We identified 13 susceptible mice and 5 resilient mice in this experiment. Compared with control mice $(n=10)$, we again did not observe differences in the density of LacZ (Fig. 9B) and cFos cells (Fig. 9C) between the three animal groups. Interestingly, we also did not find significant differences in the density of engram cells in the dorsal hippocampus between the animal groups. However, we found that susceptible mice expressed more engram cells in the ventral CA1 region than control mice (Fig. $9 D ; F_{(2,22)}=5.05 ; p=0.0156$; post hoc Tukey's test: control vs susceptible, $p=0.0259)$. When we compared the density of DAPI neurons in the CA1 of the three mouse groups, we found no difference between groups (Fig. 9E), suggesting no changes in neuronal density. Thus, enhanced engram cell formation in the dorsal hippocampus of susceptible mice was largely limited to those that respond to negative, but not neutral, stimuli. However, these findings also suggest that stress susceptibility could be related to an overall enhancement in engram cells formation for both neutral and negative stimuli in the ventral hippocampus.

Engram cell formation caused by subthreshold social defeat Exposure to chronic, but not acute, stressors is crucial for the development of depression-related behaviors (McGonagle and Kessler, 1990; McEwen, 2004). Indeed, we showed that stressing mice with only two episodes of social defeat did not result in social avoidance (Fig. 7). To find out whether this subthreshold number of defeat episodes is too weak to induce engram cell formation, we examined LacZ, cFos, and engram cell density in nonstressed mice and mice that were stressed by two episodes of social defeat (Fig. 10A). In addition to examining the SI ratio of stressed mice $1 \mathrm{~d}$ after social defeat (no delay), we examined SI ratio of another group of mice that were stressed by the subthreshold protocol $7 \mathrm{~d}$ after social defeat (with delay), which corresponds to the time point when engram cells were examined in other experiments (Fig. 1A). This control group could reveal whether engram cell density remains stable under delayed observation. Similar to what we showed in Figure 7, this subthreshold social defeat paradigm did not result in social avoidance if SI was examined $1 \mathrm{~d}$ after defeat (no delay). Surprisingly, when SI was examined $7 \mathrm{~d}$ after defeat (with delay), we found that defeated mice exhibited social avoidance (Fig. 10A; $F_{(2,22)}=5.51, p=$ 0.0115; post hoc Tukey's test: control vs defeated with delay, $p=$ 0.0142 , defeated no delay vs defeated with delay, $p=0.0494$ ). When we compared ensemble activity in these mice, we found that density of LacZ cells in both the dorsal and ventral hippocampus was significantly higher in defeated no delay mice than other mouse groups (Fig. 10B) (dorsal hippocampus: $F_{(2,21)}=$ $11.4, p=4.50 \mathrm{E}-04$; post hoc Tukey's test: control vs defeated no delay, $p=6.77 \mathrm{E}-04$, defeated no delay vs defeated with delay, $p=$ 3.27E-03; ventral hippocampus: $F_{(2,22)}=6.18, p=7.41 \mathrm{E}-03$; post hoc Tukey's test: control vs defeated no delay, $p=7.09 \mathrm{E}-03$, defeated no delay vs defeated with delay, $p=0.0475)$. The increase in LacZ cell density $1 \mathrm{~d}$ after defeat revealed the activation of CA1 neurons induced by this stressor. The decrease in LacZ cell density caused by the delay may be due to the dissipation of LacZ 
A of LacZ
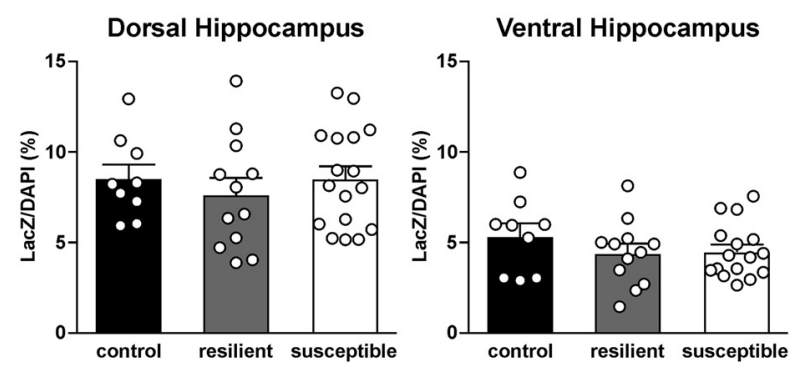

B
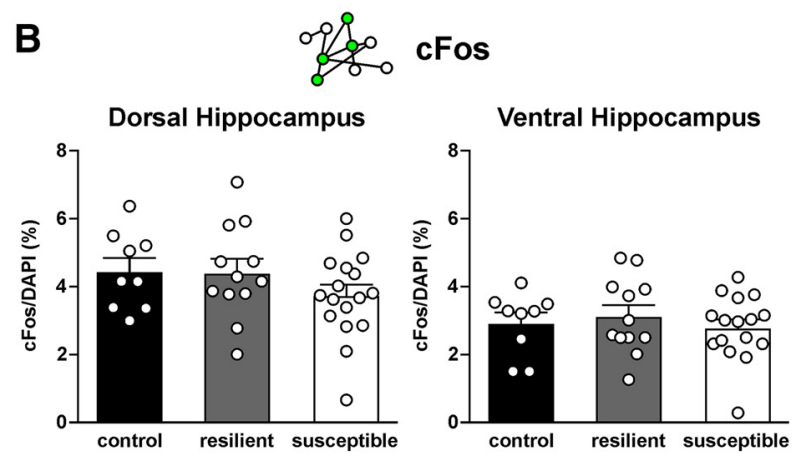

C

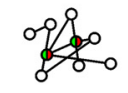

Engram cells
(LacZ+cFos)

Dorsal Hippocampus
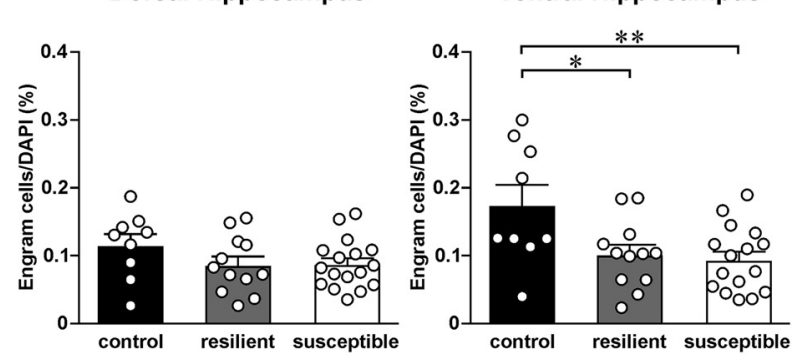

D

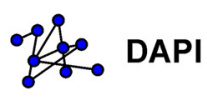

Dorsal Hippocampus

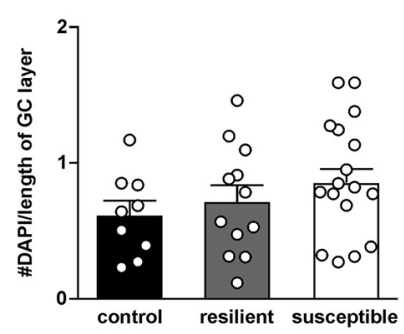

Ventral Hippocampus

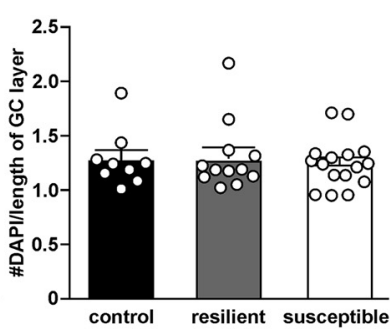

Figure 8. Expression of LacZ, CFos, and engram cells in the dentate gyrus (DG) region of the dorsal and ventral hippocampus of control, resilient, and susceptible mice. $A$, Histograms represent the density of LacZ cells in the DG region of dorsal (left) and ventral hippocampus (right) of control $(n=9)$, resilient $(n=12)$, and susceptible mice $(n=17)$. Mean \pm SEM. $\boldsymbol{B}$, Histograms represent the density of cFos cells in the DG region of dorsal (left) and ventral hippocampus (right) of control, resilient, and susceptible mice. Mean \pm SEM. C, Histograms represent the density of engram cells (double-labeled for both LacZ and (Fos) in the DG region of dorsal (left) and ventral hippocampus (right) of control, resilient, and susceptible mice. Tukey's test after ANOVA: ${ }^{*} p<0.05 ;{ }^{* *} p<0.01$. Mean \pm SEM. $\boldsymbol{D}$, Histograms represent the density of DAPI-stained cells in the DG region of dorsal (left) and ventral hippocampus (right) of control, resilient, and susceptible mice. Mean \pm SEM. signals we showed in Figure $1 D$. Unlike these changes in LacZ cells, we did not observe changes in the density of cFos (Fig. 10C), engram (Fig. 10D), and DAPI cells (Fig. 10E) between these three groups. Our findings suggest that a subthreshold social defeat paradigm does not increase the density of CA1 engram cells in the hippocampus. The expression of social avoidance after a delay from subthreshold social defeat is likely related to non-CA1 mechanisms.

\section{Discussion}

Our findings suggest that social defeat-related negative memory engrams in the hippocampal CA1 region are closely related to the expression of social avoidance in mice that are susceptible to CSDS. We found that susceptible, but not resilient, mice exhibited a higher density of CA1 engram cells than nonstressed control mice. Social avoidance not only correlated with the density of CA1 engram cells but also was facilitated and suppressed by activating and inactivating social defeat-related dorsal CA1 engram cells, respectively. Finally, a subthreshold social defeat protocol that failed to induce social avoidance did not increase engram cell density. Together, our findings suggest that the reactivation of stress-related negative memory engram cells in the CA1 region contributes to the susceptibility to CSDS.

Using social defeat to induce the labeling (LacZ by the first 2 defeat episodes) and the reactivation (cFos by an extra defeat episode $1 \mathrm{~d}$ after the SI test) of hippocampal engrams, susceptible mice showed higher CA1 engram cell reactivation than resilient and control mice. Previous findings strongly suggest that the reactivation of CA1 engram cells is related to memory retrieval that was triggered by contextual information (Deng et al., 2013; Cai et al., 2016; Roy et al., 2017). Higher engram cell density in susceptible mice therefore may be due to the facilitated retrieval of memory that is related to the defeat experience. Contextual information related to social defeat in the SI test, such as the presence of a CD1 aggressor, may be sufficient to reactivate social defeat-related engrams in susceptible mice to enhance avoidance behavior. Since pooling dorsal and ventral hippocampal data together could reveal a modest but significant increase in the density of LacZ and cFos cells in susceptible mice, stress susceptibility may also be related to enhanced hippocampal encoding of negative stimuli during CSDS. Together, increased activation and reactivation of CA1 neurons in susceptible mice make CA1 engram cell a potential cellular substrate for negative memory bias in stress-related mood disorders.

A potential mechanism to increase engram cell formation and reactivation in susceptible mice is the increase in neuronal excitability. Increase in neuronal activation could facilitate the formation of social defeat-related engrams and their reactivation. In parallel to this hypothesis, Anacker et al. (2018) have shown that social defeat can enhance the excitability of ventral hippocampal DG neurons. In addition, DG neurogenesis confers mouse resilience to CSDS by inhibiting ventral DG neuronal activity. Although we did not observe increased labeling of activated DG neurons in our study, probably due to differences in the CSDS protocols with fixed number of attacks but shorter defeat duration in the present study when compared with Anacker et al. (2018), both studies suggest the importance of high hippocampal activity to stress susceptibility. Mechanisms underlying the increased neuronal activity and excitability in susceptible mice could be due to reduced neurogenesis, as suggested by Anacker et al. (2018). However, changes in inhibitory neuronal function within the hippocampus could also be responsible. It has been shown that both parvalbumin (Morrison et al., 2016) and soma- 
A

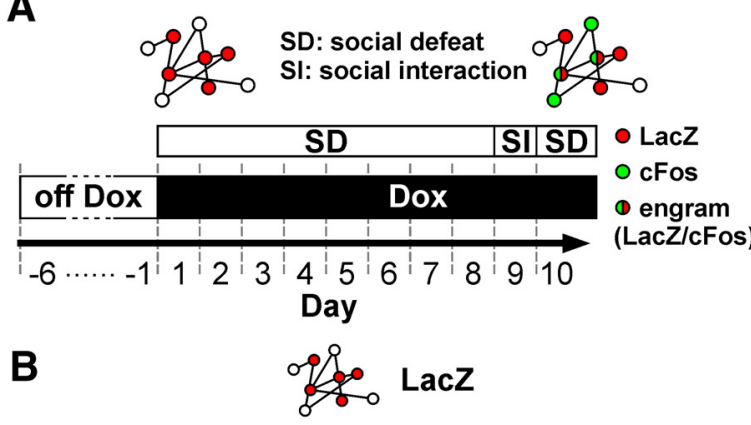

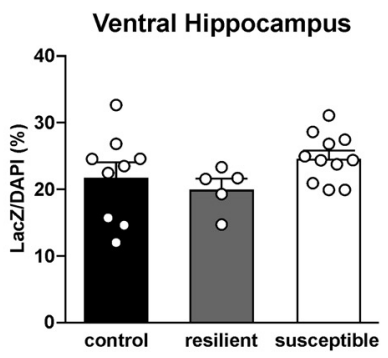

C
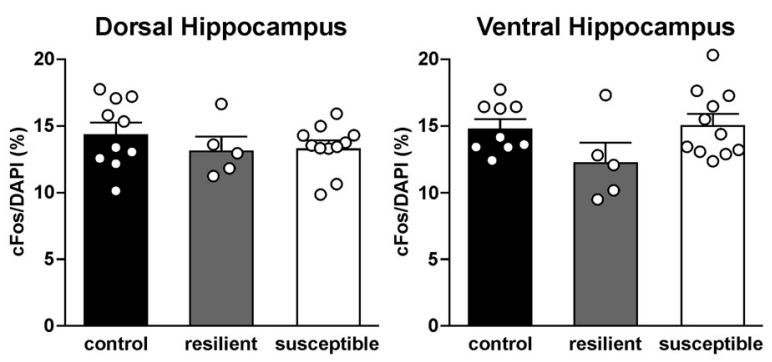

D

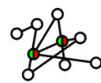

\section{Engram cells (LacZ+cFos)}

Dorsal Hippocampus
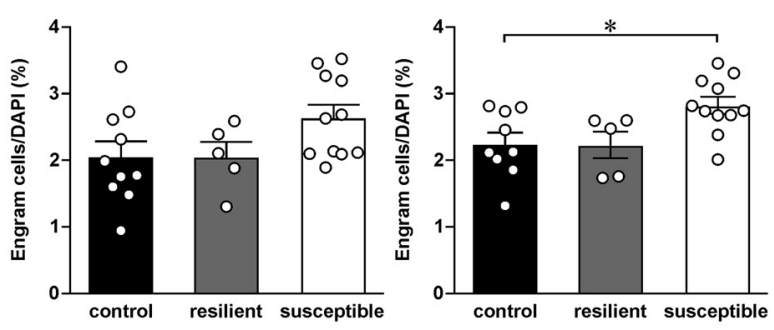

E

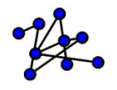

DAPI

Dorsal Hippocampus

Ventral Hippocampus
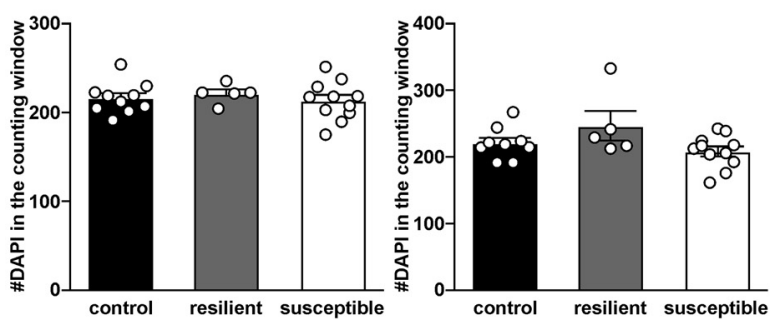

Figure 9. Expression of LaCZ, CFos, and engram cells in the CA1 region of the dorsal and ventral hippocampus of control, resilient, and susceptible mice, when labeling was stopped before social defeat. $A$, A schematic diagram of the experimental design. TetTag mice were off Dox for $4 \mathrm{~d}$. Labeling of neurons was stopped a day before the beginning of social defeat by tostatin (Stefanelli et al., 2016) expressing GABAergic neurons can regulate engram activities. One possibility is that susceptible mice show higher hippocampal activity because of reduced interneuron function. Stress has been shown to decrease the number and function of parvalbumin neurons in the hippocampus (Czeh et al., 2005). Reduced inhibitory neuronal function in the hippocampus could be an intrinsic mechanism for enhancing CA1 neuronal excitability and engram formation in both the dorsal and ventral hippocampus.

Resilient mice may be able to cope with CSDS by suppressing negative memory engram reactivation. Retrieval of mood congruent memory, which is commonly found in depression, has been suggested to reduce the ability of depressed patients for problem solving and sparing attention to positive information and memory (Conway and Pleydell-Pearce, 2000). Persistent recall of negative memory underlies rumination, which has been associated with the vulnerability to depression and the severity of depression symptoms (Alloy et al., 1999; Rude et al., 2003; Abela and Hankin, 2011) and, perhaps most importantly, hippocampal activation (Denson et al., 2009; Mandell et al., 2014). Resilient mice may be able to suppress negative memory engram reactivation through a top-down inhibitory control from the frontal lobe (Disner et al., 2011; Kircanski et al., 2012).

Ventral hippocampal activation has been proposed to underlie the susceptibility to CSDS, so that enhanced and suppressed ventral hippocampal activity could confer to the expression of susceptibility phenotypes (Anacker et al., 2018) and stress resilience (Bagot et al., 2015), respectively. Ventral hippocampal CA1 neurons bidirectionally connect to the amygdala (Petrovich et al., 2001; Cenquizca and Swanson, 2007), which plays an important role in fear learning. The ventral CA1 is also known for its roles in social memory (Okuyama et al., 2016) and social information processing (Rao et al., 2019). Enhanced excitability of ventral CA1 neurons in susceptible mice may underlie the expression of social avoidance after aversive experience, such as CSDS. In addition, the ventral CA1 projects directly to the shell of the nucleus accumbens (Groenewegen et al., 1996) and various hypothalamic nuclei (Watts et al., 1987; Kishi et al., 2000), which are known for their regulation of reward processing and circadian regulation, respectively. Enhanced ventral CA1 activity may underlie anhedonia (Krishnan et al., 2007) and sleep disturbance in susceptible mice (Wells et al., 2017).

Our findings suggest the dorsal hippocampus also regulates stress susceptibility. Both dorsal and ventral CA1 neurons show more CA1 engram cells in susceptible mice. Stress susceptibility

feeding TetTag mice with doxycycline-containing food. Mice were then stressed by 8 episodes of social defeat (SD). The interaction between TetTag mice and a CD1 mouse, the strain of aggressive mice used for SD, was examined in a social interaction (SI) test. One day after the SI test, mice underwent one more episode of SD to trigger neuronal activation. Mice were killed $90 \mathrm{~min}$ after the last episode of social defeat. Diagrams above the experimental plan represent the labeling of activated neurons during the first $2 \mathrm{~d}$ of chronic SD (red, LacZ), during the last episode of SD (green, CFos), and engram cells that expressed both signals (red/green). $\boldsymbol{B}$, Histograms represent the density of LacZ cells in the CA1 region of dorsal (left) and ventral hippocampus (right) of control $(n=10)$, resilient $(n=5)$, and susceptible mice $(n=11)$. Mean \pm SEM. C, Histograms represent the density of cFos cells in the CA1 region of dorsal (left) and ventral hippocampus (right) of control, resilient, and susceptible mice. Mean \pm SEM. D, Histograms represent the density of engram cells (double-labeled for both LacZ and CFos) in the CA1 region of dorsal (left) and ventral hippocampus (right) of control, resilient, and susceptible mice. Tukey's test after ANOVA: ${ }^{*} p<0.05$. Mean \pm SEM. E, Histograms represent the density of DAPI cells in the CA1 region of dorsal (left) and ventral hippocampus (right) of control, resilient, and susceptible mice. Mean \pm SEM. 
A

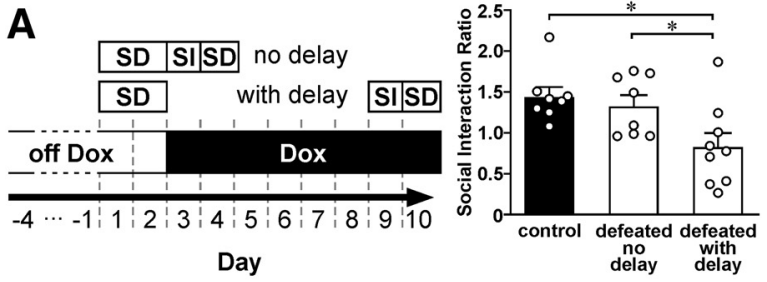

B
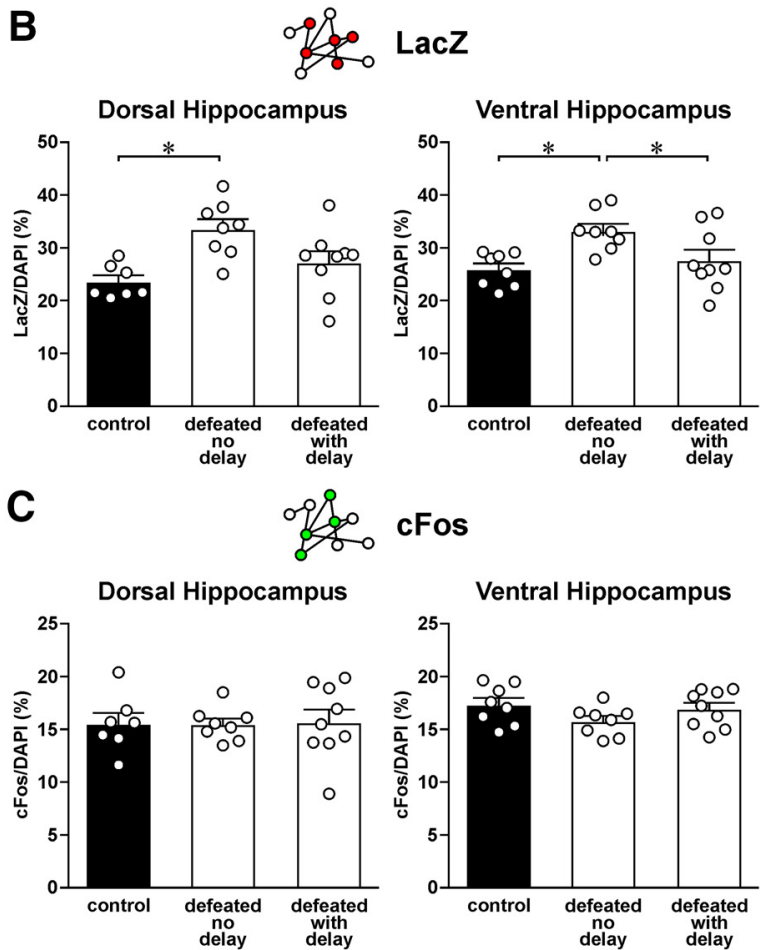

D

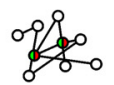

\section{Engram cells (LacZ+cFos)}

Dorsal Hippocampus
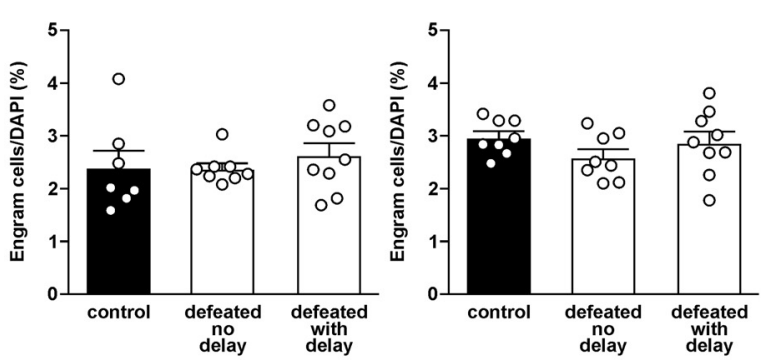

E

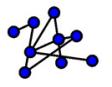

\section{DAPI}

Dorsal Hippocampus

Ventral Hippocampus
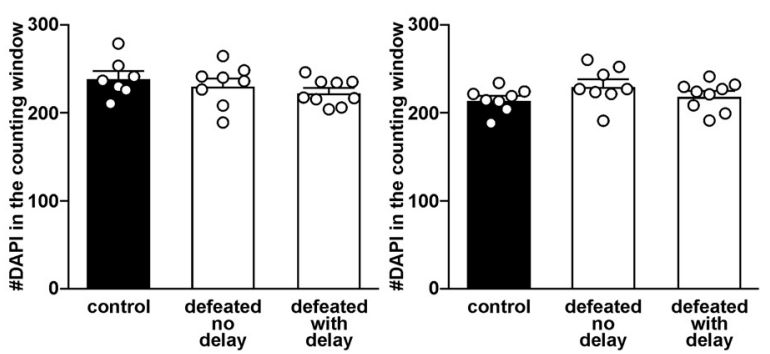

Figure 10. Expression of LacZ, CFos, and engram cells in the CA1 region of the dorsal and ventral hippocampus of mice that were stressed by a subthreshold social defeat (SD) protocol.A, Left: A schematic diagram of the experimental design. TetTag mice were off Dox for $4 \mathrm{~d}$. Some has been associated with alterations in microRNA expression (Muñoz-Llanos et al., 2018) and microstructural alterations in the dorsal hippocampus (Liu et al., 2018). We have recently shown that the dorsal hippocampus expresses fewer NMDA receptors in the extrasynaptic location (Tse et al., 2019). Activating dorsal hippocampal extrasynaptic NMDA receptors alone can reduce social avoidance in defeated mice. Cognitive factors, such as social defeat-related contextual information, are important for the expression of social avoidance. For instance, defeated mice exhibited lower levels of avoidance if an anesthetized aggressive mouse or a non-CD1 strain mouse was used in an SI test (Krishnan et al., 2007; Venzala et al., 2012). The dorsal hippocampus, which is important for associative learning of contextual fear, may contribute to stress susceptibility by encoding contextual information during social defeat. Our findings from manipulating dorsal hippocampal engram cell activity support this hypothesis.

We found that susceptible mice show higher engram cell density in the dorsal hippocampus when they were exposed to negative stimuli only (Fig. 9). This bias in engram formation cannot be found in the ventral hippocampus, where enhanced neutral and negative stimuli-related engrams were found in susceptible mice. What causes the dorsal ventral differences in neutral stimuli-related engram cells in susceptible mice remains unclear. Although the ventral hippocampus is known for its roles in emotional control, it also participates in contextual encoding. Using Arc or cFos to tag activated neurons, behavior-driven activation of ventral hippocampal neurons was observed after spatial exploration (Chawla et al., 2018), radial arm maze (Vann et al., 2000), Morris water maze (Gusev et al., 2005), and recognition memory tasks (Beer et al., 2014). Notably, significantly fewer ventral hippocampal neurons than dorsal hippocampal neurons were activated in these studies. Ventral hippocampal neurons may provide a coarse representation of space compared with dorsal hippocampal neurons, which are essential for precise spatial navigation by showing smaller firing fields and greater spatial resolution (Jung et al., 1994). It is possible that an increase in neuronal excitability in susceptible mice is sufficient to reactivate coarsely encoded contextual information in the ventral hippocampus, but not precisely encoded contextual information in the dorsal hippocampus. Alternatively, the importance of the ventral hippocampus in emotional control may facilitate the crosstalk between the processing of neutral and negative stimuli, so that

mice were stressed by two episodes of SD. Other mice were only handled when stressed mice were defeated. Labeling was blocked by putting mice on Dox-containing food after defeat. The interaction between TetTag mice and a CD1 mouse was examined in a social interaction (SI) test on $1 \mathrm{~d}$ (no delay) or $7 \mathrm{~d}$ (with delay) after SD. One day after the SI test, mice underwent one more episode of SD to trigger neuronal activation. Mice were killed $90 \mathrm{~min}$ after the last episode of SD. Right: Histograms represent the social interaction ratio of nonstressed control $(n=7)$ and defeated mice when SI tests were done either $1 \mathrm{~d}$ (no delay, $n=8$ ) or $7 \mathrm{~d}$ (with delay, $n=9$ ) after SD. Tukey's test after ANOVA: ${ }^{*} p<0.05$. Mean \pm SEM. $\boldsymbol{B}$, Histograms represent the density of LacZ cells in the CA1 region of dorsal (left) and ventral hippocampus (right) of nonstressed control and defeated mice when SI tests were done either $1 \mathrm{~d}$ (no delay) or $7 \mathrm{~d}$ (with delay) after SD. Tukey's test after ANOVA: ${ }^{*} p<0.05$. Mean \pm SEM. C, Histograms represent the density of cFos cells in the CA1 region of dorsal (left) and ventral hippocampus (right) of nonstressed control and defeated mice when SI tests were done either $1 \mathrm{~d}$ (no delay) or $7 \mathrm{~d}$ (with delay) after SD. Mean \pm SEM. $\boldsymbol{D}$, Histograms represent the density of engram cells (doublelabeled for both LacZ and (Fos) in the CA1 region of dorsal (left) and ventral hippocampus (right) of nonstressed control and defeated mice when $\mathrm{SI}$ tests were done either $1 \mathrm{~d}$ (no delay) or $7 \mathrm{~d}$ (with delay) after SD. Mean \pm SEM. E, Histograms represent the density of DAPI cells in the CA1 region of dorsal (left) and ventral hippocampus (right) of nonstressed control and defeated mice when SI tests were done either $1 \mathrm{~d}$ (no delay) or $7 \mathrm{~d}$ (with delay) after SD. Mean \pm SEM. 
neurons that are activated by general arousal or context exposure can be used to encode stress cues during social defeat.

Our findings that subthreshold social defeat failed to increase hippocampal CA1 engram cell density could have several implications. We found that stressed mice at $1 \mathrm{~d}$ after the subthreshold defeat protocol exhibited a significant increase in CA1 LacZ cell density, but no change in the SI ratio (Fig. 10, the defeated no delay group), suggesting that, without the formation of engram cells, high CA1 neuronal activation in both the dorsal and ventral hippocampus alone is not sufficient to induce social avoidance. When social behavior of a separate group of mice stressed by subthreshold social defeat was examined a week after defeat, we observed social avoidance but no change in the density of CA1 LacZ, cFos, and engram cells. It remains unclear why social avoidance occurs after a delay. The lack of engram cell formation in mice that show delayed expression of social avoidance suggests the involvement of non-CA1 mechanisms. Given the well-known effect of time to generalize fear memory (Wiltgen and Silva, 2007), the delayed expression of social avoidance in mice stressed by subthreshold social defeat may be due to fear generalization. However, we found that examining the density of LacZ, cFos, and engram cells in the DG, which plays important roles in fear generalization (Yokoyama and Matsuo, 2016), revealed no differences in engram cell density between animal groups (dorsal hippocampus: $F_{(2,22)}=0.408, p=0.670$; ventral hippocampus: $\left.F_{(2,21)}=0.221, p=0.804\right)$. Alternatively, the need of a delay for the expression of a fear response may be related to fear incubation. For instance, electric shocks and cues pairing have revealed an increase in fear responses at 1 and 2 months compared with $2 \mathrm{~d}$ after conditioning (Pickens et al., 2009). Although social avoidance was observed at 1 month after CSDS (Krishnan et al., 2007), whether an incubation effect of time to worsen avoidance after CSDS remains to be determined. Future studies could also examine whether changes in neuronal activation in nonhippocampal regions that are involved in fear incubation (e.g., medial amygdala) (Tsuda et al., 2015) or fear generalization (central amygdala) (Botta et al., 2015); nucleus reuniens and PFC (Xu and Südhof, 2013) are responsible for the delayed expression of social avoidance after a subthreshold social defeat. Finally, our findings suggest that a chronic stressor, which is closely associated to the etiology of depression (McGonagle and Kessler, 1990; McEwen, 2004), rather than a short subthreshold stressor, is needed to induce changes in engram cells. Persistent plastic changes at the synaptic and cellular levels may be induced by repeated stress exposure to facilitate the reactivation of engram cells in susceptible mice.

Unlike the CA1 region, we did not find differences in DG engrams between susceptible and resilient mice. Instead, we observed lower engram cell density in stressed mice compared with nonstressed control mice. DG plays important roles in pattern separation and is likely sensitive to changes in context (Leutgeb et al., 2007). Using TetTag mice, it has been shown that, while engram cells were formed in both the CA1 and DG during contextual learning, subsequent exposure to the same context favored the reactivation of CA1, but not DG engram cells (Deng et al., 2013). Since mice were kept in a similar context for multiple days during CSDS, CA1 instead of DG ensembles may be preferentially reactivated under this behavioral paradigm. Indeed, compared with 4\%-9\% reactivation of DG cells in a relatively short behavioral task, such as fear conditioning (Liu et al., 2012; Denny et al., 2014; Stefanelli et al., 2016), only $\sim 0.5 \%$ of DG cells were reactivated by social defeat in the current study.
Similar to a recent report using the same mouse model (Deng et al., 2013), we were not able to detect CA3 engram cells in TetTag mice due to low LacZ expression in this hippocampal region (Fig. 3). In the experiment for detecting the duration of LacZ expression after social defeat, we found a large number of LacZ cells in the CA3 region $1 \mathrm{~d}$ after social defeat. However, LacZ signals seemed to disappear quickly in the CA3 region so that almost no LacZ cells were found in the pyramidal layer of the CA3 region at $4 \mathrm{~d}$ after social defeat. Indeed, we saw high levels of cFos expression in the $\mathrm{CA} 3$ region during reactivation of $\mathrm{CA} 1$ and DG engram cells, suggesting the activation of $\mathrm{CA} 3$ cells during memory recall. It is unclear why long-term LacZ expression can be found in the CA1 and DG regions, but not in the CA3 region. Since LacZ expression is sustained by the tetracycline-insensitive tTA after the reintroduction of Dox food, the lack of CA3 LacZ signal may be due to poor expression of this mutated tTA in the $\mathrm{CA} 3$ region. The role of CA3 engrams in stress susceptibility cannot be ruled out because CA3 engrams have been shown to be more sensitive to fear-related contextual information than neutral novel context (Denny et al., 2014). Using the Cre-dependent ArcCreER ${ }^{\mathrm{T} 2}$ mouse line may reveal the contribution of CA3 neurons to stress susceptibility.

The difference in negative memory engrams between susceptible and resilient mice has important implications for depression. Changes in these memory functions could be related to the bottom-up changes from a hypersensitive medial temporal lobe, including hyperfunctioning of the amygdala and the hippocampus. Our findings that negative memory engrams are increased in mice that are susceptible to CSDS suggested that these engrams could mechanistically contribute to the negative bias of memory formation in depression. Negative memory engrams correlated with the expression of social avoidance, suggesting their roles in mediating cognitive symptoms of depression. Inhibiting negative memory engrams in the hippocampus could be a novel therapeutic approach for treating cognitive symptoms in depression.

\section{References}

Abela JR, Hankin BL (2011) Rumination as a vulnerability factor to depression during the transition from early to middle adolescence: a multiwave longitudinal study. J Abnorm Psychol 120:259-271.

Alloy LB, Abramson LY, Whitehouse WG, Hogan ME, Tashman NA, Steinberg DL, Rose DT, Donovan P (1999) Depressogenic cognitive styles: predictive validity, information processing and personality characteristics, and developmental origins. Behav Res Ther 37:503-531.

Anacker C, Luna VM, Stevens GS, Millette A, Shores R, Jimenez JC, Chen B, Hen R (2018) Hippocampal neurogenesis confers stress resilience by inhibiting the ventral dentate gyrus. Nature 559:98-102.

Bagot RC, Parise EM, Peña CJ, Zhang HX, Maze I, Chaudhury D, Persaud B, Cachope R, Bolaños-Guzmán CA, Cheer JF, Cheer J, Deisseroth K, Han MH, Nestler EJ (2015) Ventral hippocampal afferents to the nucleus accumbens regulate susceptibility to depression. Nat Commun 6:7062.

Beer Z, Chwiesko C, Sauvage MM (2014) Processing of spatial and nonspatial information reveals functional homogeneity along the dorsoventral axis of CA3, but not CA1. Neurobiol Learn Mem 111:56-64.

Botta P, Demmou L, Kasugai Y, Markovic M, Xu C, Fadok JP, Lu T, Poe MM, Xu L, Cook JM, Rudolph U, Sah P, Ferraguti F, Lüthi A (2015) Regulating anxiety with extrasynaptic inhibition. Nat Neurosci 18:1493-1500.

Cai DJ, Aharoni D, Shuman T, Shobe J, Biane J, Song W, Wei B, Veshkini M, La-Vu M, Lou J, Flores SE, Kim I, Sano Y, Zhou M, Baumgaertel K, Lavi A, Kamata M, Tuszynski M, Mayford M, Golshani P, et al. (2016) A shared neural ensemble links distinct contextual memories encoded close in time. Nature 534:115-118.

Cenquizca LA, Swanson LW (2007) Spatial organization of direct hippocampal field CA1 axonal projections to the rest of the cerebral cortex. Brain Res Rev 56:1-26.

Chawla MK, Sutherland VL, Olson K, McNaughton BL, Barnes CA (2018) Behavior-driven arc expression is reduced in all ventral hippocampal sub- 
fields compared to CA1, CA3, and dentate gyrus in rat dorsal hippocampus. Hippocampus 28:178-185.

Conway MA, Pleydell-Pearce CW (2000) The construction of autobiographical memories in the self-memory system. Psychol Rev 107:261-288.

Czeh B, Simon M, van der Hart MG, Schmelting B, Hesselink MB, Fuchs E (2005) Chronic stress decreases the number of parvalbumin-immunoreactive interneurons in the hippocampus: prevention by treatment with a substance P receptor (NK1) antagonist. Neuropsychopharmacology 30:6779.

Deng W, Mayford M, Gage FH (2013) Selection of distinct populations of dentate granule cells in response to inputs as a mechanism for pattern separation in mice. Elife 2:e00312.

Denny CA, Kheirbek MA, Alba EL, Tanaka KF, Brachman RA, Laughman KB, Tomm NK, Turi GF, Losonczy A, Hen R (2014) Hippocampal memory traces are differentially modulated by experience, time, and adult neurogenesis. Neuron 83:189-201.

Denson TF, Pedersen WC, Ronquillo J, Nandy AS (2009) The angry brain: neural correlates of anger, angry rumination, and aggressive personality. J Cogn Neurosci 21:734-744.

Disner SG, Beevers CG, Haigh EA, Beck AT (2011) Neural mechanisms of the cognitive model of depression. Nat Rev Neurosci 12:467-477.

El Iskandrani KS, Oosterhof CA, El Mansari M, Blier P (2015) Impact of subanesthetic doses of ketamine on AMPA-mediated responses in rats: an in vivo electrophysiological study on monoaminergic and glutamatergic neurons. J Psychopharmacol 29:792-801.

Fanselow MS, Dong HW (2010) Are the dorsal and ventral hippocampus functionally distinct structures? Neuron 65:7-19.

Fu CH, Williams SC, Cleare AJ, Brammer MJ, Walsh ND, Kim J, Andrew CM, Pich EM, Williams PM, Reed LJ, Mitterschiffthaler MT, Suckling J, Bullmore ET (2004) Attenuation of the neural response to sad faces in major depression by antidepressant treatment: a prospective, event-related functional magnetic resonance imaging study. Arch Gen Psychiatry 61: 877-889.

Gaddy MA, Ingram RE (2014) A meta-analytic review of mood-congruent implicit memory in depressed mood. Clin Psychol Rev 34:402-416.

Groenewegen HJ, Wright CI, Beijer AV (1996) The nucleus accumbens: gateway for limbic structures to reach the motor system? Prog Brain Res 107:485-511.

Gusev PA, Cui C, Alkon DL, Gubin AN (2005) Topography of Arc/Arg3.1 mRNA expression in the dorsal and ventral hippocampus induced by recent and remote spatial memory recall: dissociation of CA3 and CA1 activation. J Neurosci 25:9384-9397.

Hamilton JP, Gotlib IH (2008) Neural substrates of increased memory sensitivity for negative stimuli in major depression. Biol Psychiatry 63: $1155-1162$

Hertel PT, Gerstle M (2003) Depressive deficits in forgetting. Psychol Sci 14:573-578

Joormann J, Quinn ME (2014) Cognitive processes and emotion regulation in depression. Depress Anxiety 31:308-315.

Josselyn SA, Köhler S, Frankland PW (2015) Finding the engram. Nat Rev Neurosci 16:521-534.

Jung MW, Wiener SI, McNaughton BL (1994) Comparison of spatial firing characteristics of units in dorsal and ventral hippocampus of the rat. J Neurosci 14:7347-7356.

Kircanski K, Joormann J, Gotlib IH (2012) Cognitive aspects of depression. Wiley Interdiscip Rev Cogn Sci 3:301-313.

Kishi T, Tsumori T, Ono K, Yokota S, Ishino H, Yasui Y (2000) Topographical organization of projections from the subiculum to the hypothalamus in the rat. J Comp Neurol 419:205-222.

Koster EH, De Raedt R, Leyman L, De Lissnyder E (2010) Mood-congruent attention and memory bias in dysphoria: exploring the coherence among information-processing biases. Behav Res Ther 48:219-225.

Krishnan V, Han MH, Graham DL, Berton O, Renthal W, Russo SJ, Laplant Q, Graham A, Lutter M, Lagace DC, Ghose S, Reister R, Tannous P, Green TA, Neve RL, Chakravarty S, Kumar A, Eisch AJ, Self DW, Lee FS, et al. (2007) Molecular adaptations underlying susceptibility and resistance to social defeat in brain reward regions. Cell 131:391-404.

Leutgeb JK, Leutgeb S, Moser MB, Moser EI (2007) Pattern separation in the dentate gyrus and CA3 of the hippocampus. Science 315:961-966.

Liu X, Ramirez S, Pang PT, Puryear CB, Govindarajan A, Deisseroth K, Tonegawa S (2012) Optogenetic stimulation of a hippocampal engram activates fear memory recall. Nature 484:381-385.
Liu X, Yuan J, Guang Y, Wang X, Feng Z (2018) Longitudinal in vivo diffusion tensor imaging detects differential microstructural alterations in the hippocampus of chronic social defeat stress-susceptible and resilient mice. Front Neurosci 12:613.

Lyubomirsky S, Caldwell ND, Nolen-Hoeksema S (1998) Effects of ruminative and distracting responses to depressed mood on retrieval of autobiographical memories. J Pers Soc Psychol 75:166-177.

Maeng S, Zarate CA Jr, Du J, Schloesser RJ, McCammon J, Chen G, Manji HK (2008) Cellular mechanisms underlying the antidepressant effects of ketamine: role of alpha-amino-3-hydroxy-5-methylisoxazole-4-propionic acid receptors. Biol Psychiatry 63:349-352.

Mandell D, Siegle GJ, Shutt L, Feldmiller J, Thase ME (2014) Neural substrates of trait ruminations in depression. J Abnorm Psychol 123:35-48.

Mayberg HS, Brannan SK, Tekell JL, Silva JA, Mahurin RK, McGinnis S, Jerabek PA (2000) Regional metabolic effects of fluoxetine in major depression: serial changes and relationship to clinical response. Biol Psychiatry $48: 830-843$.

McEwen BS (2004) Protection and damage from acute and chronic stress: allostasis and allostatic overload and relevance to the pathophysiology of psychiatric disorders. Ann N Y Acad Sci 1032:1-7.

McGonagle KA, Kessler RC (1990) Chronic stress, acute stress, and depressive symptoms. Am J Community Psychol 18:681-706.

McKinnon MC, Yucel K, Nazarov A, MacQueen GM (2009) A metaanalysis examining clinical predictors of hippocampal volume in patients with major depressive disorder. J Psychiatry Neurosci 34:41-54.

Morrison DJ, Rashid AJ, Yiu AP, Yan C, Frankland PW, Josselyn SA (2016) Parvalbumin interneurons constrain the size of the lateral amygdala engram. Neurobiol Learn Mem 135:91-99.

Muñoz-Llanos M, Garcia-Perez MA, Xu X, Tejos-Bravo M, Vidal EA, Moyano TC, Gutiérrez RA, Aguayo FI, Pacheco A, García-Rojo G, Aliaga E, Rojas PS, Cidlowski JA, Fiedler JL (2018) MicroRNA profiling and bioinformatics target analysis in dorsal hippocampus of chronically stressed rats: relevance to depression pathophysiology. Front Mol Neurosci 11:251.

Nolen-Hoeksema S (2000) The role of rumination in depressive disorders and mixed anxiety/depressive symptoms. J Abnorm Psychol 109:504511.

Okuyama T, Kitamura T, Roy DS, Itohara S, Tonegawa S (2016) Ventral CA1 neurons store social memory. Science 353:1536-1541.

Petrovich GD, Canteras NS, Swanson LW (2001) Combinatorial amygdalar inputs to hippocampal domains and hypothalamic behavior systems. Brain Res Brain Res Rev 38:247-289.

Pickens CL, Golden SA, Adams-Deutsch T, Nair SG, Shaham Y (2009) Long-lasting incubation of conditioned fear in rats. Biol Psychiatry 65: 881-886.

Radulovic J, Kammermeier J, Spiess J (1998) Relationship between fos production and classical fear conditioning: effects of novelty, latent inhibition, and unconditioned stimulus preexposure. J Neurosci 18:7452-7461.

Ramirez S, Liu X, MacDonald CJ, Moffa A, Zhou J, Redondo RL, Tonegawa S (2015) Activating positive memory engrams suppresses depression-like behaviour. Nature 522:335-339.

Rao RP, von Heimendahl M, Bahr V, Brecht M (2019) Neuronal responses to conspecifics in the ventral CA1. Cell Rep 27:3460-3472.e3.

Reijmers LG, Perkins BL, Matsuo N, Mayford M (2007) Localization of a stable neural correlate of associative memory. Science 317:1230-1233.

Roy DS, Kitamura T, Okuyama T, Ogawa SK, Sun C, Obata Y, Yoshiki A, Tonegawa S (2017) Distinct neural circuits for the formation and retrieval of episodic memories. Cell 170:1000-1012.e19.

Rude SS, Valdez CR, Odom S, Ebrahimi A (2003) Negative cognitive biases predict subsequent depression. Cognit Ther Res 27:415-429.

Santarelli L, Saxe M, Gross C, Surget A, Battaglia F, Dulawa S, Weisstaub N, Lee J, Duman R, Arancio O, Belzung C, Hen R (2003) Requirement of hippocampal neurogenesis for the behavioral effects of antidepressants. Science 301:805-809.

Sørensen AT, Cooper YA, Baratta MV, Weng FJ, Zhang Y, Ramamoorthi K, Fropf R, LaVerriere E, Xue J, Young A, Schneider C, Gøtzsche CR, Hemberg M, Yin JC, Maier SF, Lin Y (2016) A robust activity marking system for exploring active neuronal ensembles. Elife 5:e13918.

Squire LR (1992) Memory and the hippocampus: a synthesis from findings with rats, monkeys, and humans. Psychol Rev 99:195-231.

Stefanelli T, Bertollini C, Lüscher C, Muller D, Mendez P (2016) Hip- 
pocampal somatostatin interneurons control the size of neuronal memory ensembles. Neuron 89:1074-1085.

Thomas EJ, Elliott R, McKie S, Arnone D, Downey D, Juhasz G, Deakin JF, Anderson IM (2011) Interaction between a history of depression and rumination on neural response to emotional faces. Psychol Med 41: $1845-1855$.

Tonegawa S, Liu X, Ramirez S, Redondo R (2015) Memory engram cells have come of age. Neuron 87:918-931.

Tse YC, Montoya I, Wong AS, Mathieu A, Lissemore J, Lagace DC, Wong TP (2014) A longitudinal study of stress-induced hippocampal volume changes in mice that are susceptible or resilient to chronic social defeat. Hippocampus 24:1120-1128.

Tse YC, Lopez J, Moquin A, Wong SA, Maysinger D, Wong TP (2019) The susceptibility to chronic social defeat stress is related to low hippocampal extrasynaptic NMDA receptor function. Neuropsychopharmacology 44:1310-1318.

Tsuda MC, Yeung HM, Kuo J, Usdin TB (2015) Incubation of fear is regulated by TIP39 peptide signaling in the medial nucleus of the amygdala. J Neurosci 35:12152-12161.

Vann SD, Brown MW, Erichsen JT, Aggleton JP (2000) Fos imaging reveals differential patterns of hippocampal and parahippocampal subfield activation in rats in response to different spatial memory tests. J Neurosci 20:2711-2718.

Venzala E, Garcia-Garcia AL, Elizalde N, Delagrange P, Tordera RM (2012) Chronic social defeat stress model: behavioral features, antidepressant action, and interaction with biological risk factors. Psychopharmacology (Berl) 224:313-325.

Videbech P, Ravnkilde B (2004) Hippocampal volume and depression: a meta-analysis of MRI studies. Am J Psychiatry 161:1957-1966.

Watts AG, Swanson LW, Sanchez-Watts G (1987) Efferent projections of the suprachiasmatic nucleus: I. Studies using anterograde transport of phaseolus vulgaris leucoagglutinin in the rat. J Comp Neurol 258: 204-229.

Wells AM, Ridener E, Bourbonais CA, Kim W, Pantazopoulos H, Carroll FI, Kim KS, Cohen BM, Carlezon WA Jr (2017) Effects of chronic social defeat stress on sleep and circadian rhythms are mitigated by kappaopioid receptor antagonism. J Neurosci 37:7656-7668.

Wiltgen BJ, Silva AJ (2007) Memory for context becomes less specific with time. Learn Mem 14:313-317.

Xu W, Südhof TC (2013) A neural circuit for memory specificity and generalization. Science 339:1290-1295.

Yokoyama M, Matsuo N (2016) Loss of ensemble segregation in dentate gyrus, but not in somatosensory cortex, during contextual fear memory generalization. Front Behav Neurosci 10:218.

Zhang Z, Ferretti V, Güntan I, Moro A, Steinberg EA, Ye Z, Zecharia AY, Yu X, Vyssotski AL, Brickley SG, Yustos R, Pillidge ZE, Harding EC, Wisden W, Franks NP (2015) Neuronal ensembles sufficient for recovery sleep and the sedative actions of alpha2 adrenergic agonists. Nat Neurosci 18 : 553-561. 